\title{
Consenso Gallego Multidisciplinar sobre el Uso de Hipolipemiantes
}

\author{
Galician multidisciplinary consensus \\ about the use of lipid-lowering drugs
}

\author{
Botana López $\mathrm{MA}^{1}$, Aneiros Díaz $\mathrm{AM}^{9}$, Bouza Piñeiro $\mathrm{P}^{8}$, Caamaño Isorna $C^{3}$, Castro Iglesias $\mathrm{A}^{10}$, \\ Díaz Díaz $\mathrm{JL}^{7}$, Lago Deibe $\mathrm{Fl}^{2}$, Pérez Freijomil $\mathrm{A}^{6}$, Rey Aldana $\mathrm{D}^{4}$, Vázquez Rodríguez $\mathrm{JM}^{5}$ \\ 1 Sociedad Gallega de Endocrinología, Nutrición y Metabolismo \\ ${ }^{2}$ Asociación Galega de Medicina Familiar e Comunitaria (AGAMFEC) \\ ${ }^{3}$ Sociedad Española de Farmacéuticos de Atención Primaria \\ ${ }^{4}$ Sociedad Española de Médicos de Atención Primaria - Galicia (SEMERGEN-Galicia) \\ ${ }^{5}$ Sociedad Gallega de Cardiología \\ ${ }^{6}$ Sociedad Gallega de Médicos Generales y de Familia (SEMG Galicia) \\ ${ }^{7}$ Sociedad Gallega de Medicina Interna \\ ${ }^{8}$ Sociedad Gallega de Nefrología \\ ${ }^{9}$ Sociedad Gallega de Neurología \\ ${ }^{10}$ Sociedad Gallega Interdisciplinaria del SIDA
}

\section{Introducción}

Las enfermedades cardiovasculares (ECV) constituyen la principal causa de morbilidad y mortalidad mundial ${ }^{1}$. Cada año fallecen más personas por ECV que por cualquier otra causa. Se calcula que en 2012 murieron por ECV 17,5 millones de personas, lo cual representa un $30 \%$ de todos los decesos registrados en el mundo?

Según datos del INE sobre las causas de mortalidad del año 2013, la enfermedad cardiovascular sigue manteniéndose como la primera causa de defunción en España $(30,1 \%)$ por encima del cáncer $(28,44 \%)$ y de las enfermedades respiratorias $(10,9 \%)$. Por comunidades autónomas, Galicia (33,73\%), Andalucía (32,99\%) y Asturias (32,81\%) son las que cuentan con un porcentaje de mortalidad por causa cardiovascular más elevado, mientras que Canarias (22,85\%), Madrid $(26,30 \%)$ y País Vasco $(27,48 \%)$ son las de menor mortalidad cardiovascular.

Estas pérdidas humanas suponen también un daño económico sustancial que se calcula en torno al $2 \%$ del PIB tanto para Estados Unidos como para Europa ${ }^{3}$.

La ECV está fuertemente relacionada con el estilo de vida, en especial con el consumo de tabaco, hábitos de vida poco saludables, la inactividad física y el estrés psicosocial ${ }^{4}$. Según la OMS, con cambios adecuados del estilo de vida se podrían prevenir más de tres cuartas partes de la mortalidad cardiovascular ${ }^{5}$.

Los estudios epidemiológicos muestran una clara relación entre las cifras de colesterol total en suero y el riesgo cardiovascular6. El consumo de hipolipemiantes en España ha pasado de 18,9 dosis diarias por mil habitantes día (DHD) en el año 2000 a 102,6 DHD en el año 2012, lo que supone un incremento del $442 \%{ }^{7}$. Las estatinas son los fármacos más utilizados, repre- sentando un 89,3\% del consumo de hipolipemiantes en 2012 . Las estatinas han sido el grupo de hipolipemiantes más consumidos a lo largo de todo el periodo de estudio, con 14,7 DHD en 2000 y 91,6 DHD en 2012 (incremento de 522\%). El fármaco más utilizado durante todo el periodo de estudio fue la atorvastatina, que ha pasado de 3,8 DHD en 2000 a 42,8 DHD en 2012 (incremento de 1012\%). También hay que destacar la simvastatina como segunda estatina más consumida a lo largo de este periodo, con 3,4 DHD en 2000 y 29,6 DHD en 2012. Atorvastatina y simvastatina representaron en 2012 el 78,2\% del consumo del total de estatinas ${ }^{7}$. El consumo de fibratos ha disminuido respecto al consumo total de hipolipemiantes del 19,4\% en 2000 al 5,9\% en 2012 ${ }^{7}$. El resto de hipolipemiantes ("otros hipolipemiantes") representaron el 4,8\% del consumo total en 2012, destacando ezetimiba y ácidos grasos omega $3^{7}$.

Según datos internos del SERGAS en 2014 la atorvastatina fue el segundo principio activo más prescrito después del omeprazol, representando un 3,33\% de todos los envases dispensados y un 2,44\% de la facturación total del SERGAS. Las guías Europeas para el manejo de la Dislipemia y Prevención Cardiovascular, publicadas respectivamente en 2011 y 2012 por un comité conjunto de ESC/EAS (European Society of Cardiology/European Atherosclerosis Society) $)^{8,9}$ han tenido buena acogida en España, con diversas iniciativas para fomentar su aplicación. A finales del 2013, se publicó la guía del American College of Cardiology/American Heart Association (ACC/AHA) ${ }^{10}$ que inmediatamente ha generado polémica a ambos lados del Atlántico. Su abandono de las concentraciones de colesterol como objetivo a alcanzar y su visión de las estatinas como fármacos que reducen el riesgo cardiovascular rompió con déca- 
das de recomendaciones. Él método del cálculo de riesgo de las guías y los umbrales de tratamiento recomendados han sido criticados casi de inmediato. Según la revista JAMA su aplicación supondría 923 millones de personas a tratamiento con estatinas (estatinización de la sociedad) ${ }^{11}$.

En medio de esta polémica, este grupo de trabajo es partidario de seguir manteniendo objetivos de tratamiento en función de las concentraciones de cLDL, a excepción de determinadas situaciones como en la enfermedad renal crónica. En Julio de 2014 National Institute for Health and Care Excellence (NICE) publicó una nueva actualización de su guía de práctica clínica de prevención primaria y secundaria de la enfermedad cardiovascular ${ }^{12}$. Dicha publicación ha avivado más el debate a nivel internacional. Los aspectos más controvertidos son la disminución del umbral de riesgo de personas sanas y favorecer el uso de atorvastatina frente otras alternativas. Con las nuevas recomendaciones, el número de pacientes a tratamiento se incrementaría en 4,5 millones de personas en el Reino Unido ${ }^{12}$.

Los perfiles de riesgo y la importancia de los factores de riesgo difieren entre las poblaciones y debemos ser muy cuidadosos a la hora de aplicar a una sociedad recomendaciones elaboradas para otra.. Debemos evitar las extrapolaciones. Experiencias anteriores muestran que herramientas previas como las guías de cálculo de riesgo Framingham y de la ATP III (Third Adult Treatment Panel) ${ }^{13}$ se adaptaron y adoptaron de forma extensa en todo el mundo.

Además, el estudio sobre la seguridad de los pacientes en atención primaria en España (APEAS), establece que el $48,2 \%$ de los efectos adversos observados están relacionados con la medicación ${ }^{14}$.

En respuesta a esta situación se presenta este documento, cuyo propósito es proporcionar información científica, objetiva, completa y actualizada a los profesionales sanitarios para ayudarles en la toma de decisiones en diferentes situaciones, con dos objetivos claros: el beneficio del paciente y la sostenibilidad del sistema sanitario.

\section{Definición de objetivos de tratamiento}

La aplicación exclusivamente de medidas preventivas poblacionales para el control de las enfermedades cardiovasculares no es un objetivo real. Por consiguiente, la identificación del paciente en alto riesgo es esencial para iniciar medidas preventivas más intensas y optimizar la relación coste-efectividad de los tratamientos hipolipemiantes.

En las últimas guías de práctica clínica ESC/EAS sobre el manejo de las dislipemias, se aconseja estimar el riesgo cardiovascular mediante el sistema SCORE, que está basado en series de datos de cohortes europeas, representativas de nuestra población. Este sistema estima el riesgo a 10 años de un primer evento arterioesclerótico mortal ${ }^{1,2}$.

El sistema SCORE presenta limitaciones. Por un lado los pacientes jóvenes, que pueden tener un riesgo absoluto bajo, pero que presentan un riesgo relativo elevado (riesgo eleva- do a largo plazo). Por este motivo, son de especial utilidad las tablas de riesgo relativo específicas para este colectivo ${ }^{1,2}$. Por otro lado, los pacientes mayores, fundamentalmente los varones, que solo por la edad tendrían una estimación de riesgo absoluto superior al 50 al 10\% según las tablas SCO$\mathrm{RE}$, lo que puede conducir a sobretratar a este colectivo.

Por otra parte, si queremos conocer el riesgo que tiene un paciente de sufrir un evento cardiovascular no mortal, se estima que éste es 3 veces mayor al riesgo obtenido por SCORE. Debemos establecer el nivel de riesgo cardiovascular de cada paciente y determinar, en función del mismo, un objetivo terapéutico en base al c-LDL según la ESC/EAS'. De esta forma identificaremos los siguientes grupos de riesgo:

\section{Riesgo muy alto:}

- Enfermedad cardiovascular establecida.

- DM1 o DM2 con más de un factor de riesgo cardiovascular o lesión de órgano diana.

- Enfermedad renal crónica (ERC) grave (TFG $<30 \mathrm{ml} /$ $\left.\min / 1,73 \mathrm{~m}^{2}\right)$.

- SCORE $\geq 10 \%$.

\section{Riesgo alto:}

- Factores de riesgo específicos marcadamente elevados, como dislipemia familiar aterogénica e hipertensión grave.

- DM1 o DM2, pero sin factores de riesgo cardiovascular, ni lesión de órgano diana.

- ERC moderada (TFG 30-59 ml/min/1,73 m²).

- SCORE $\geq 5 \%$ y $<10 \%$.

\section{Riesgo moderado:}

- SCORE $\geq 1 \%$ y $<5 \%$.

\section{Riesgo bajo:}

- SCORE $<1 \%$.

No existe un consenso absoluto sobre cuál debe ser el objetivo de tratamiento entre las diferentes guías de práctica clínica ${ }^{2,3,4,5}$. La guía ESC/EAS continúa definiendo los objetivos terapéuticos según los niveles de colesterol unido a lipoproteínas de baja densidad (C-LDL), basada en los resultados del Cholesterol Treatment Trialists Collaboration $(\mathrm{CTT})^{6,7}$, que confirmó que la reducción de la enfermedad cardiovascular depende fundamentalmente de la disminución de las cifras de c-LDL. Una reducción de c-LDL de $1 \mathrm{mmol} / \mathrm{l}$ ( 40 mg/dL) se asocia con un descenso estimado del $20 \%$ en los eventos cardiovasculares.

Según estas guías, los objetivos terapéuticos son los siguientes:

- Para pacientes con un riesgo cardiovascular bajo y pacientes con riesgo moderado, el colesterol plasmático total deber ser $<5 \mathrm{mmo} / / \mathrm{l}(190 \mathrm{mg} / \mathrm{dL})$ y el $\mathrm{CLDL},<3$ $\mathrm{mmol} / \mathrm{l}(115 \mathrm{mg} / \mathrm{dL})$.

- Pacientes con riesgo alto, el objetivo terapéutico recomendado es llegar a cifras de cLDL $<100 \mathrm{mg} / \mathrm{dL}$ $(<2,5 \mathrm{mmol} / \mathrm{l})$. 
- Pacientes de muy alto riesgo, el objetivo terapéutico es alcanzar cifras de $\mathrm{CLDL}<70 \mathrm{mg} / \mathrm{dL}(<1,8 \mathrm{mmol} / \mathrm{l})$ 0 una reducción $\geq 50 \%$ del valor inicial.

La modificación del estilo de vida es un pilar fundamental del tratamiento del paciente con dislipemia y para el manejo del riesgo cardiovascular total. Debemos recomendar a todos los pacientes las siguientes modificaciones:

- La dieta debe ser variada, con una ingesta calórica adaptada a cada individuo para evitar el sobrepeso y la obesidad.

- Se promoverá el consumo de fruta, verduras, legumbres, frutos secos, cereales, panes integrales y pescado (especialmente azul)

- La grasa saturada se sustituirá por los alimentos mencionados antes y por grasas monoinsaturadas y poliinsaturadas de origen vegetal.

- La ingesta de sal debe reducirse a menos de $5 \mathrm{~g} / \mathrm{día}$, evitando el consumo de sal de mesa.

- Recomendaremos moderación en el consumo de alcohol (<10-20 g/día a las mujeres y <20-30 g/día a los varones); los pacientes con hipertrigliceridemia deben abstenerse de consumir alcohol.

- Debe limitarse la ingesta de bebidas (especialmente refrescos) y alimentos con azúcar añadido, especialmente los pacientes con hipertrigliceridemia.

- Debe promoverse la actividad física con el objetivo de alcanzar la práctica de ejercicio regular durante al menos $30 \mathrm{~min} / \mathrm{d}$ ía todos los días.

- Debe evitarse el consumo y la exposición a productos del tabaco.

\section{Tratamiento de las dislipemias en prevención primaria}

En prevención primaria, la promoción de estilos de vida saludable a través de la dieta y la actividad física y el abandono del tabaco deben permanecer como actividades preferentes. En prevención primaria las estatinas, al cabo de 5 años, reducen de forma moderada la mortalidad total así como los eventos coronarios y cardiovasculares, los ictus y las revascularizaciones ${ }^{1}$. El metaanálisis del grupo Cholesterol Treatment Trialists Collaborators $(\mathrm{CTT})^{2}$ concluye que la evidencia apoya el beneficio de las estatinas en prevención primaria, independientemente de la edad, sexo o nivel de c-LDL basal. Este análisis ha sido criticado por cuestiones metodológicas. Muchos de los grandes ensayos clínicos con estatinas adolecen de un sesgo de selección y los efectos adversos graves tampoco se recogieron siempre de forma adecuada.

Por tanto, en las personas de riesgo cardiovascular bajo el beneficio es escaso, lo que unido a la posibilidad de efectos adversos a largo plazo hace que la relación beneficio/riesgo probablemente no sea tan favorable.

A finales de 2013 se actualizaron las guías americanas ATP$\mathrm{IV}^{4}$, que ya han sido comentadas y con las que este grupo de trabajo no está completamente de acuerdo.
Como ya se ha comentado las nuevas guías NICE ${ }^{5}$ también incrementan de forma significativa la necesidad de tratamiento. Recomiendan:

- Discutir con el paciente los beneficios que supone la modificación del estilo de vida y ofrecerle la oportunidad de volver a evaluar su riesgo después de que han tratado de cambiar su estilo de vida. Si la modificación del estilo de vida resulta ineficaz o inapropiada después de la evaluación de riesgos, ofrecer tratamiento con estatinas.

- Ofrecer atorvastatina $\mathbf{2 0 ~} \mathrm{mg} 0$ equivalente para la prevención primaria de las enfermedades cardiovasculares a las personas que tienen un riesgo de desarrollar ECV a los 10 años de 10\% o mayor (estimado con QRISK2: http://www.heartscore.org/Pages/ welcome.aspx).

- En las personas con diabetes tipo 1: ofrecer estatinas para la prevención primaria a los pacientes de más de 40 años y con un tiempo de evolución de la diabetes superior a 10 años, así como a aquellos con nefropatía establecida 0 con otros factores de riesgo $\mathrm{CV}$.

- En las personas con diabetes tipo 2: ofrecer estatinas para la prevención primaria a las personas con diabetes tipo 2 que tienen un riesgo $\mathrm{CV}^{3} 10 \%$ (estimado con QRISK2).

El Comité Español Interdisciplinario de Prevención Cardiovascular y la Sociedad Española de Cardiología aconseja (enero de 2015) seguir usando las guías europeas de prevención cardiovascular de 2012 basadas en SCORE, pues tienen una mejor evidencia que las anteriores. Las poblaciones a las que se refieren las guías americanas y NICE son muy diferentes de las españolas, y su riesgo cardiovascular es 3-4 veces superior por lo que tienen una dudosa aplicabilidad en nuestro medio.

La guía europea ofrece unas pautas de estratificación del riesgo mucho mejor validadas que la propuesta estadounidense y la sugerida por la NICE evitando así un riesgo evidente de tratamiento farmacológico innecesario en prevención primaria, sobre todo en personas mayores. Creemos que sus mensajes son más adecuados para el entorno español y previenen un posible sobretratamiento en prevención primaria.

\section{Uso de hipolipemiantes en cardiopatía isquémica y arteriopatía periférica (prevención secundaria)}

El cLDL es el indicador de respuesta al tratamiento más utilizado en los ensayos clínicos que han analizado los efectos de la terapia hipolipemiante en pacientes con enfermedad cardiovascular conocida. Se ha estimado que una reducción de $40 \mathrm{mg} / \mathrm{dl}$ en la concentración de cLDL se acompaña de una reducción del $22 \%$ en la morbimortalidad cardiovascular ${ }^{1}$.

En los pacientes de muy alto riesgo de un evento coronario (los pacientes con cardiopatía isquémica) el objetivo recomendado 
Tabla 1. Posicionamiento y recomendaciones del Comité Español Interdisciplinario de Prevención Cardiovascular y la Sociedad Española de Cardiología sobre manejo y tratamiento de las dislipemias.

\begin{tabular}{|c|c|c|}
\hline $\begin{array}{l}\text { Prevención primaria } \\
\text { Riesgo alto: } S C O R E \geq 5 \\
\text { - Un solo factor de riesgo muy elevado (p.e. dislipemias } \\
\text { familiares, hipertensión arterial grave) } \geq 180 \text { sistólica } 0 \\
\geq 110 \text { diastólica, pacientes } \\
\text { con cifras aisladas de colesterol superiores a 320mg/ } \\
\text { dL y/o a } 240 \mathrm{mg} / \mathrm{dL} \text { de c-LDL. } \\
\text { - DM1 o DM2 sin otros FRCV ni daño } \\
\text { de órgano diana. } \\
\text { - Enfermedad renal crónica moderada } \\
\text { (FGe, } 30-59 \mathrm{ml} / \mathrm{min} / 1,73 \mathrm{~m}^{2} \text { ). }\end{array}$ & $\begin{array}{l}\text { Objetivo terapéutico recomendado: } \\
\mathrm{cLDL}<100 \mathrm{mg} / \mathrm{dL}(<2,5 \mathrm{mmol} / \mathrm{l}) \text {. }\end{array}$ & $\begin{array}{l}\text { No existe evidencia sólida que respalde los umbrales o } \\
\text { puntos de corte para definir las categorías de riesgo. } \\
\text { El balance riesgo-beneficio y el coste-efectividad } \\
\text { del tratamiento con estatinas es menos claro cuanto } \\
\text { menor es el riesgo cardiovascular basal. }\end{array}$ \\
\hline $\begin{array}{l}\text { Riesgo moderado (SCORE } \geq 1-<5 \% \text { ). } \\
\text { Riesgo bajo (SCORE }<1 \% \text { ). }\end{array}$ & $\begin{array}{l}\text { Objetivo terapéutico recomendado: } \\
\text { - colesterol total }<190 \mathrm{mg} / \mathrm{dL} \\
\text { - cLDL }<115 \mathrm{mg} / \mathrm{dL}\end{array}$ & \multirow{2}{*}{$\begin{array}{l}\text { Las tablas deben estar basadas en población } \\
\text { autóctona. } \\
\text { La guía estadounidense incluye poblaciones } \\
\text { de características y riesgos muy diferentes } \\
\text { a la población española. }\end{array}$} \\
\hline $\begin{array}{l}\text { Calculadoras para la estimación } \\
\text { del riesgo cardiovascular. }\end{array}$ & $\begin{array}{l}\text { En España se sigue recomendado el uso de las tablas } \\
\text { europeas SCORE para países de bajo riesgo }\end{array}$ & \\
\hline Empleo de fármacos distintos de las estatinas. & $\begin{array}{l}\text { Considerar inhibidores de la absorción } \\
\text { como ezetimiba o resinas, además de estatinas, } \\
\text { como tratamiento combinado } \\
\text { para lograr los objetivos de cLDL. } \\
\text { La hipertrigliceridemia y/o el cHDL bajo son factores } \\
\text { de riesgo independientes a tener en cuenta. Requieren } \\
\text { una modificación intensa del estilo de vida y } \\
\text { considerar fármacos (fibratos) si persisten. Otra opción } \\
\text { para reducir los triglicéridos son los ácidos grasos } \\
\text { omega-3. }\end{array}$ & $\begin{array}{l}\text { La hipertrigliceridemia moderada se asocia con mayor } \\
\text { riesgo cardiovascular que la más grave (triglicéridos > } \\
900 \mathrm{mg} / \mathrm{dL} \text { ), que es un factor de riesgo de pancreatitis } \\
\text { que requiere tratamiento per se (fibratos). }\end{array}$ \\
\hline
\end{tabular}

Tabla 2. (tomada de Clin Invest Arteriosc. 2012; 24:30-39).

Tabla 3 Porcentaje de reducción de colesterol unido a lipoproteinas de baja densidad (CLDL) de las estatinas

\begin{tabular}{|c|c|c|c|c|c|c|c|}
\hline Estatina & $20.25 \%$ & $26-30 \%$ & $31.35 \%$ & $36.40 \%$ & $41-508$ & $51.55 \%$ & $56.60 \%$ \\
\hline Pravastatina ${ }^{a}$ & $10 \mathrm{mg}$ & $20 \mathrm{mg}$ & $40 \mathrm{mg}$ & & & & \\
\hline Fluvastatina & $20 \mathrm{~ms}$ & $40 \mathrm{~ms}$ & $80 \mathrm{~ms}$ & & & & \\
\hline Lovastatina & $10 \mathrm{mg}$ & $20 \mathrm{mg}$ & $40 \mathrm{mg}$ & 80 & & & \\
\hline Simvastatina & & $10 \mathrm{mg}$ & $20 \mathrm{mg}$ & $40 \mathrm{mg}$ & $80 \mathrm{mg}$ & & \\
\hline Atorvastatina & & & $10 \mathrm{mg}$ & $20 \mathrm{mg}$ & $40 \mathrm{mg}$ & $80 \mathrm{mg}$ & \\
\hline Rosuvastatina ${ }^{a}$ & & & & $5 \mathrm{mg}$ & $10 \mathrm{mg}$ & $20 \mathrm{mg}$ & $40 \mathrm{mg}^{\mathrm{b}}$ \\
\hline Pitavastatina & & & $1 \mathrm{mg}$ & $2 \mathrm{mg}$ & $4 \mathrm{mg}$ & & \\
\hline
\end{tabular}

Los miembros del grupo que ha elaborado este consenso creen que esta tabla es la que mejor refleja las características comparativas de las diferentes estatinas que actualmente están disponibles en el mercado español. Los datos de la tabla han sido obtenidos de estudios de eficacia individual de las estatinas y no de comparaciones entre ellas. Las dosis más elevadas de simvastatina y de rosuvastatina no son recomendadas. Considerar la asociación de ezetimiba cuando sea preciso llegar a dichas dosis.

por la Sociedad Europea de Cardiología es una concentración de cLDL menor 0 igual a $70 \mathrm{mg} / \mathrm{dl} 0$ una reducción mayor del $50 \%$ de los valores previos. Si dudamos de la estimación del cLDL podemos utilizar como objetivo el colesterol no HDL (diferencia entre el colesterol total y $\mathrm{CHDL}$ ) que ha de ser menor o igual a $100 \mathrm{mg} / \mathrm{dl}$ en los pacientes de muy alto riesgo?.

En los pacientes con arteriopatía periférica se considera indicado el tratamiento con estatinas para mantener un CLDL inferior a $100 \mathrm{mg} / \mathrm{dl}$, aunque de manera óptima debería establecerse un objetivo de CLDL menor de $70 \mathrm{mg} / \mathrm{dl}^{3}$.

Los pacientes con un síndrome coronario agudo deben ser tratados con estatinas a dosis altas durante su hospitalización, independientemente de los niveles de colesterol. En los pacientes con antecedentes de IAM se recomienda el tratamiento farmacológico aún cuando el cLDL basal sea inferior a $70 \mathrm{mg} / \mathrm{dl}^{4}$. El estudio IMPROVE-IT ${ }^{5}$ ha mostrado que la asociación de ezetimibe (10 mg/dia) y simvastatina (40 mg/dia) en pacien- tes que han sufrido un síndrome coronario agudo en los últimos diez días, produce una mayor reducción de colesterol LDL y de eventos cardiovasculares cuando se compara con simvastatina (40 mg/dia) en monoterapia.

La arteriopatía periférica que afecta a las arterias de los miembros inferiores se considera equivalente a la enfermedad coronaria y es indicación de tratamiento hipolipemiante. No está claro el beneficio del tratamiento hipolipemiante en pacientes con aneurisma de la aorta abdominal ni arteriopatía en otras localizaciones (ej., en las arterias retinianas).

\section{Prevención secundaria en el ictus isquémico}

La reducción de los niveles de colesterol en sangre ha resultado beneficiosa en la prevención secundaria del Ictus isquémi$\mathrm{co}$, siendo menos evidente en la prevención primaria ${ }^{1,2}$.

Los niveles de CLDL se asocian de forma independiente al riesgo de ictus ${ }^{3}$. Otras fracciones lipídicas también se aso- 
cian a riesgo vascular; los triglicéridos, cHDL y lipoproteína a. Diversos estudios en prevención secundaria no han demostrado eficacia en combinaciones de estatinas con niacina, ni del uso de fármacos que aumentan $\mathrm{CHDL}^{4,5}$.

Las estatinas ejercen un efecto independiente de la reducción de la concentración CLDL, llamado efecto pleiotrópico, sobre la pared vascular. La reducción del grosor íntima-media, que se relaciona directamente con riesgo vascular aterotrombótico, es mayor con estatinas a altas dosis 6 . También favorecen al aumento del óxido nítrico (vasodilatación), tienen efecto antioxidante, antiexcitotóxico, favorecen la disolución del trombo y promueven la angiogénesis ${ }^{7,8}$.

En la fase aguda del ictus las estatinas favorecen la recuperación en todos los tipos de ictus isquémico, siendo mayor si se usan dosis altas ${ }^{9}$.

Se han realizado numerosos estudios de prevención secundaria en pacientes con eventos cardíacos, si bien hay un único estudio prospectivo y aleatorizado sobre estatinas e ictus, el estudio SPARCL, publicado en el año $2006^{10}$. Demostró que, tras sufrir un ictus isquémico o AlT, la toma de $80 \mathrm{mg} / \mathrm{día}$ de atorvastatina reducía en un $16 \%$ el riesgo de ictus frente a placebo. Esta disminución se produjo a expensas de una reducción del $43 \%$ en la probabilidad de presentar un ictus fatal, y resultó no significativa para el ictus no fatal. Este beneficio se mantuvo los 5 años que duró el seguimiento. La mortalidad global y cardiovascular no fue diferente entre ambos grupos, con un aumento de ictus hemorrágicos no fatales en el grupo de estatinas (2.3\% vs $1.4 \%)$. Se evitaría un nuevo ictus isquémico por cada 53 pacientes tratados durante 5 años, sin reducción de la mortalidad, a expensas de sufrir un ictus hemorrágico por cada 112 pacientes tratados. En análisis posteriores ${ }^{11}$ se ha observado un mayor beneficio en pacientes con estenosis carotídea, se mantiene el beneficio y seguridad en pacientes mayores de 65 años y no se detectó mayor riesgo de hemorragia con niveles bajos de CLDL.

Se recomienda, por tanto, el uso de estatinas en la fase aguda del ictus isquémico o AIT, independientemente de su causa (pequeño vaso, gran vaso o cardioembólico).

En prevención secundaria recomendamos el uso de atorvastatina a dosis de $80 \mathrm{mg} / \mathrm{d}$, disminuyendo la dosis en caso de riesgo de interacción farmacológica, efectos adversos 0 por preferencia del paciente. A partir de los 6 meses del tratamiento se recomienda evaluar el objetivo de reducción de $>40 \%$ del nivel inicial de colesterol LDL ${ }^{12}$.

El objetivo de reducción de cLDL en prevención secundaria del ictus isquémico/AlT es alcanzar niveles de $<70 \mathrm{mg} / \mathrm{dl}$ o la reducción de al menos el $50 \%$ con respecto a las cifras basales.

\section{Tratamiento farmacológico hipolipemiante en ancianos}

Como en pacientes más jóvenes, antes de plantear el tratamiento hipolipemiante en ancianos, es importante considerar la existencia de causas secundarias, que deberán ser corregidas cuando sea posible, como son las enfermedades renales y hepáticas, el hipotiroidismo, así como el uso de determinados fármacos como algunos hipotensores o antipsicóticos ${ }^{1}$.

Las estatinas han demostrado de manera consistente que reducen el riesgo de eventos y muertes coronarias en prevención secundaria ${ }^{2}$. Datos del estudio SPARCL, realizado en pacientes con ictus o AIT reciente, también demostraron la eficacia de estatinas en la reducción de ictus en mayores de 65 años $^{3}$. En prevención primaria los datos no son tan concluyentes, aunque un metaanálisis específicamente realizado en pacientes mayores de 65 años en PP concluye que el tratamiento con estatinas disminuye los ictus (NNT 42 en un año) e infartos (NNT 24 en un año) pero no la mortalidad cardiovascular ni la total ${ }^{4}$.

A partir de 80-85 años las pruebas para tratar este grupo de edad son escasas. Se usará el juicio clínico, ponderando la relación riesgo/beneficio junto con los deseos del paciente ${ }^{5}$. La ausencia de datos para pacientes mayores en las tablas de riesgo $\mathrm{CV}$ hace que sea muy importante considerar la presencia de datos de arteriopatía subclínica, como el aumento del grosor de la intima media de la carótida, la presencia de calcio coronario o la reducción del índice tobillo brazo, lo que orientaría la decisión a favor del tratamiento farmacológico ${ }^{6}$.

A considerar también en los ancianos es la calidad y expectativa de vida. Se precisan al menos dos años para que el tratamiento con estatinas logre beneficios, por lo que los pacientes con una probable supervivencia menor no deberían ser tratados ${ }^{1}$, especialmente pacientes en cuidados paliativos, demencias avanzadas, cáncer, insuficiencia renal 0 cardiaca avanzadas, enfermedades pulmonares avanzadas y conectivopatías ${ }^{7}$. Igualmente los ancianos frágiles, que no han sido incluidos en los ensayos clínicos, se excluirán del tratamiento dada la mayor probabilidad de efectos secundarios y que no se ha demostrado reducción de eventos, al menos en los institucionalizados sin enfermedad cardiovascular ${ }^{1}$. Otros factores a considerar en pacientes ancianos son el deterioro cognitivo y la comorbilidad, ambos factores relacionados negativamente con el cumplimiento ${ }^{6}$, así como la polifarmacia ${ }^{3}$, por el aumento de riesgo de efectos secundarios, sobre todo miopatía, ${ }^{8,9}$.

Los ancianos son más sensibles a los efectos secundarios de los fármacos, por los cambios farmacocinéticos y farmacodinámicos que sufren, pero los datos demuestran que las estatinas son razonablemente seguras, aunque una prescripción prudente aconseja iniciar dosis menores e incrementarlas paulatinamente hasta lograr el objetivo terapéutico $0^{9,10,11,12}$. De manera resumida ancianos en prevención secundaria se deben tratar igual que los más jóvenes; en prevención primaria se tratan si el RCV es alto o muy alto, dado que reduce eventos aunque no muertes. Para simplificar el cálculo, probablemente por el límite superior de edad en la tabla SCORE, la Guía Europea ${ }^{10}$ aconseja hacerlo cuando coexiste al menos otro factor de RCV mayor, titulando la dosis por el riesgo de efectos secundarios. El principal problema se da en los muy ancianos (80 o más años), ahí se ha propuesto recientemente en España un algoritmo para indicar estatinas ${ }^{4,13}$. 
De los fármacos no estatinas, casi no hay datos en ancianos por lo que su uso debe considerarse excepcional. Datos del IMPROVE IT sugieren que el beneficio de la asociación de ezetimiba y simvastatina se mantiene en mayores de 75 años ${ }^{14}$ aunque el hecho de que dicho estudio haya sido analizado a 7 años implica la necesidad de valorar la esperanza de vida de forma individual.

\section{Uso de hipolipemiantes en Diabetes Mellitus}

La diabetes mellitus, fundamentalmente la tipo 2, comporta un aumento de riesgo de mortalidad cardiovascular (RCV) de entre 2 y 4 veces. En la diabetes la enfermedad arteriosclerótica es la principal causa de morbimortalidad: supone el $75 \%$ de los ingresos hospitalarios en diabéticos y el $80 \%$ de los fallecimientos en estos pacientes (75\% arteriosclerosis coronaria; $25 \%$ enfermedad cerebrovascular 0 arterial periférica) ${ }^{1}$, mientras que la insuficiencia cardíaca y la enfermedad arterial periférica son las formas iniciales de presentación más frecuente?2.

Existe una "dislipemia diabética", típica de la diabetes tipo 2, común a otras situaciones de insulin-resistencia, que cursa con CHDL reducido, hipertrigliceridemia y niveles de CLDL no especialmente elevados, pero sí muy aterogénicos ${ }^{3}$. Son partículas de CLDL más pequeñas y densas, por lo que a una determinada concentración de colesterol hay un mayor número de ellas y pasan más fácilmente a formar parte de la placa de ateroma ${ }^{3}$.

Existe una relación claramente demostrada entre el CLDL y la arteriosclerosis, relación que no está tan aclarada con el cHDL y con los triglicéridos ${ }^{4,5}$. El cLDL es un predictor muy potente de RCV en pacientes diabéticos, y en éstos se ha demostrado un aumento del $12 \%$ del RCV por cada incremento de $10 \mathrm{mg} / \mathrm{dL}$ de $\mathrm{CLDL}^{6}$.

El CLDL sigue siendo, por lo tanto, el principal objetivo de tratamiento en los individuos diabéticos a la hora de tratar el factor lipídico. La ESC (Sociedad Europea de Cardiología) / EASD (Sociedad Europea para el Estudio de la Diabetes) recomiendan reducir el CLDL a $<100$ si no existen otros factores de RCV y, si existe algún otro adicional, el objetivo sería alcanzar un $\mathrm{CLDL}<70$. Si estos valores no son alcanzables, se recomienda reducir al menos el cLDL en un $50 \%{ }^{7}$.

Las concentraciones de apolipoproteína B (ApoB) y de colesterol no HDL (noHDLC) podrían ser mejores que el CLDL como predictores de RCV en pacientes diabéticos ${ }^{8}$. Actualmente se consideran objetivos secundarios 0 alternativos porque no hay evidencia que demuestre que usarlos como objetivos terapéuticos es superior al cLDL como objetivo para la reducción de eventos CV. En personas con triglicéridos elevados el noHDLc expresa con más precisión la aterogenicidad de las lipoproteínas circulantes.

Las guías recientemente publicadas por la American College of Cardiology/American Heart Association (ACC/AHA), y asimiladas también por la American Diabetes Association (ADA), plantean la "estatinización" generalizada de todos los pacientes diabéticos entre 40 y 75 años de edad que tengan un CLDL superior a 70, con lo que denominan estatinas de moderada intensidad o de alta intensidad dependiendo del RCV global y no de los valores de cLDL conseguidos.

Por otra parte, con posterioridad ha aparecido evidencia que refuerza el mensaje de que la reducción de RCV está más en relación con el grado de reducción de cLDL y no tanto con el fármaco utilizado para conseguirlo9.

Nosotros creemos que el inicio de fármacos hipolipemiantes (fundamentalmente estatinas por su eficacia y eficiencia, pero no exclusivamente) debe hacerse en función de los objetivos de CLDL que queremos alcanzar, y su continuidad y ajuste de dosis en función de los valores de cLDL que se van consiguiendo.

Las estatinas constituyen la terapia de primera línea para el tratamiento de la dislipemia en los sujetos diabéticos. Su uso está indicado en todos los pacientes diabéticos que tengan un CLDL mayor de 1000 en aquellos que tengan un RCV muy elevado y un CLDL mayor de 70.

Dado el elevado RCV de los pacientes diabéticos, se puede utilizar la estrategia de usar la estatina más eficaz a la dosis más alta tolerada. Nosotros proponemos que la selección de la estatina y de su dosis se haga en función del porcentaje de reducción de CLDL que necesitemos (según el CLDL de "partida" y el cLDL objetivo) y la reducción predecible que se puede obtener con cada fármaco o asociación (ver tabla previa del apartado de prevención primaria).

En caso de que con la estatina no se consiga la reducción suficiente de CLDL, 0 en el caso de intolerancia a la estatina que impida llegar a la dosis deseada, se iniciará terapia combinada. El fármaco de elección a utilizar en combinación con la estatina será la ezetimiba. Alternativamente se propone el uso de resinas de intercambio.

Aunque todas las estatinas mejoran el RCV, se ha comprobado que se asocian a un mayor riesgo de desarrollo de diabetes 0 a un discreto deterioro del control metabólico ${ }^{10,11}$. No obstante este riesgo es muy inferior a la mejoría inducida por la reducción del CLDL. Probablemente la pitavastatina no tenga este aumento de riesgo de desarrollar diabetes ${ }^{12}$.

\section{Tratamiento de la dislipemia en la enfermedad renal crónica}

La enfermedad cardiovascular representa la principal causa de muerte de los pacientes con enfermedad renal crónica (ERC) ${ }^{1}$, cuyo riesgo se incrementa con el progresivo descenso del filtrado glomerular ( $F G)$. La mayoría de los enfermos con ERC estadios 3-5 (G3-G5, Tabla 3) muestran una dislipemia mixta de perfil altamente aterogénico, típicamente caracterizado por una elevación de los triglicéridos y una disminución del $\mathrm{CHDL}^{2}$.

Las actuales guías $\mathrm{KDIGO}^{3}$ prescinden del $\mathrm{CLDL}$ a la hora de decidir a qué pacientes con ERC debemos prescribir tratamiento hipolipemiante, así como de la consecución de determinados valores como objetivo del mismo, debido a la falta de evidencia sobre los beneficios reales que supone alcanzar una determinada cifra de $\mathrm{CLDL}^{4}$ en esta población. Estas recomendaciones están basadas en la utilización de estatinas ${ }^{5}$, bien solas 0 en combinación con ezetimiba ${ }^{6}$. 
Tabla 3. Clasificicación de la ERC (KDIGO 2012')

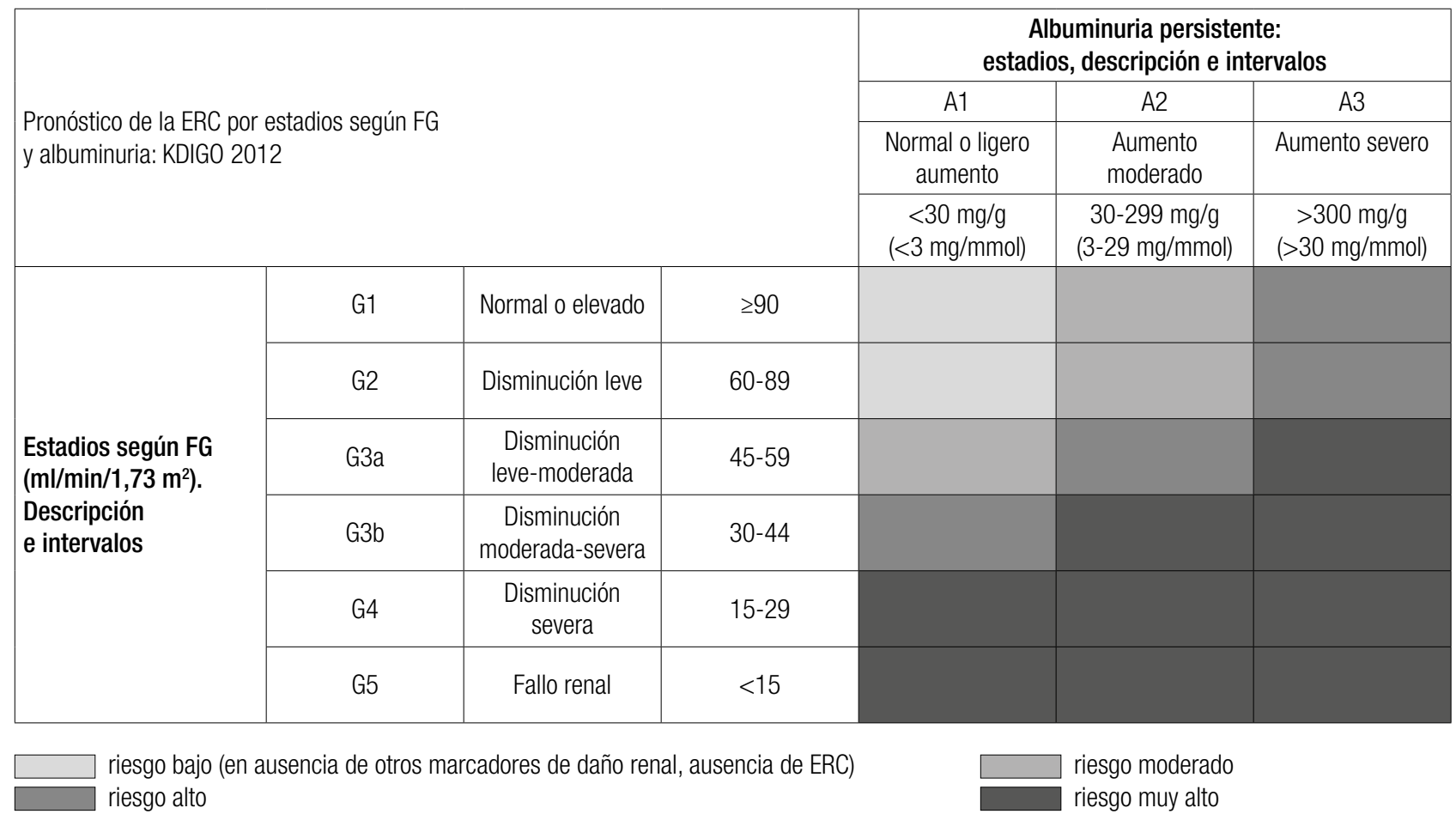

\section{Recomendaciones}

1. Realizar un perfil lipídico a todo paciente al que se detecte una ERC, así como a los pacientes en diálisis o trasplantados.

2. En los pacientes con ERC (incluidos pacientes en diálisis 0 trasplantados) no será preciso realizar determinaciones periódicas del perfil lipídico salvo que se espere realizar algún cambio terapéutico.

Se recomienda determinar el perfil lipídico en las siguientes situaciones: para valorar la adherencia al tratamiento, cambios en la modalidad de terapia renal sustitutiva, valoración de dislipemias secundarias (síndrome nefrótico, diabetes mellitus, hipotiroidismo, etc.) o para la valoración del riesgo vascular a los 10 años en pacientes de $<50$ años que no estén recibiendo ya una estatina. Se sugiere una valoración anual del riesgo vascular en la mayoría de los pacientes.

3. En adultos de $>50$ años con $F G$ estimado ( $F G e)<60 \mathrm{ml} /$ $\mathrm{min} / 1,73 \mathrm{~m}^{2}$ que no estén en tratamiento renal sustitutivo (diálisis o trasplante) se recomienda el tratamiento con estatinas, solas 0 en asociación con ezetimiba.

4. En adultos de $>50$ años con $F G e ~>60 \mathrm{ml} / \mathrm{min} / 1,73 \mathrm{~m}^{2}$ (ERC estadio G1-G2) con proteinuria o albuminuria se recomienda el tratamiento con una estatina.

5. En adultos entre los 18 y 49 años con ERC no en diálisis ni trasplantados, se recomienda el tratamiento con estatinas si cumple al menos una de las siguientes condiciones:

- Enfermedad coronaria previa (IAM o revascularización coronaria).

- Diabetes mellitus.
- ACV previo.

- Incidencia estimada a los 10 años de muerte coronaria 0 IAM no fatal $>10 \%$.

- La mayoría de estas herramientas de cálculo de RCV no consideran la existencia de ERC en sus ecuaciones por lo que su fiabilidad predictiva no está garantizada, en especial en sujetos menores de 40 años?

- A pesar de la falta de evidencia sobre los beneficios del tratamiento hipolipemiante en el síndrome nefrótico8, deberá ser considerado en pacientes jóvenes con hiperlipemia severa (ej., CLDL $\geq 190 \mathrm{mg} / \mathrm{dl}$ ) dado el elevado riesgo vascular que esta situación conlleva ${ }^{7,9}$.

6. En pacientes con ERC en diálisis no deberá iniciarse tratamiento hipolipemiante (estatinas solas o en combinación con ezetimiba), recomendando su continuidad en pacientes tratados previamente al comienzo de la diálisis.

Tanto los ensayos clínicos realizados específicamente en población en diálisis ${ }^{10,11}$ como los metaanálisis ${ }^{12}$ no han permitido demostrar un beneficio de la terapia con estatinas, por lo que no se recomienda iniciar su uso salvo que el paciente ya lo estuviese recibiendo previamente ${ }^{6}$.

7. En adultos receptores de un trasplante renal se recomienda el tratamiento con estatinas.

Resultados inciertos de las estatinas sobre la disminución de episodios cardiovasculares y mortalidad global ${ }^{13,14}$, así como sobre la supervivencia del injerto ${ }^{14}$. Debido a las interacciones con los anticalcineurínicos (ciclosporina y tacrolimus) se recomienda iniciar el tratamiento con dosis bajas, especialmente en el caso de la ciclosporina ${ }^{7}$. 


\section{Seguridad de las estatinas en la ERC}

En etapas iniciales de la ERC (estadios G1 y G2, FGe $>60$ $\mathrm{ml} / \mathrm{min} / 1,73 \mathrm{~m}^{2}$ ) no es preciso realizar ajustes en su dosificación. Sin embargo, en fases más avanzadas (estadios G3G5) existen diferencias en el perfil farmacocinético que pueden suponer una ventaja en cuanto a su seguridad ${ }^{15}$. En este sentido, la utilización de estatinas de eliminación hepática (fluvastatina, atorvastatina y pitavastatina) podría disminuir el riesgo de efectos adversos, así como restringir el uso en pacientes polimedicados de aquellas metabolizadas a través de citocromo P450-3A4 (lovastatina, simvastatina y atorvastatina $^{16}$. Con estas precauciones cualquier estatina podría, en principio, ser prescrita, recomendándose no obstante el uso de aquellas estatinas y dosis que hayan mostrado beneficios en los ensayos clínicos realizados específicamente en estas poblaciones de pacientes ${ }^{3}$ (Tabla 4).

\section{Tratamiento de la dislipemia en el paciente trasplantado}

La dislipemia es una complicación frecuente en el postrasplante ${ }^{1}$, jugando el tratamiento inmunosupresor un papel fundamental en el desarrollo de la misma. De los fármacos habitualmente utilizados como terapia de mantenimiento, los que inducen una mayor alteración lipídica son, por orden decreciente: los inhibidores de mTOR (sirolimus y everolimus), los esteroides y los inhibidores de la calcineurina (ciclosporina y tacrolimus, especialmente la primera). Los antimetabolitos (azatioprina y micofenolato) carecen de efecto hiperlipemiante? En trasplantados cardíacos, los estudios aleatorizados han mostrado resultados favorables de las estatinas sobre la supervivencia de los pacientes y sobre la aparición de vasculopatía del injerto ${ }^{3,4,5}$, por lo que se recomienda su introducción temprana tras el trasplante independientemente de las cifras de colesterol ${ }^{6}$. Las estatinas han mostrado además efectos inmunomoduladores y antitumorales ${ }^{7,8,9}$, recomendándose también su uso en otros trasplantes de órganos sólidos, en especial en pacientes con historia de enfermedad cardiovascular. En trasplantados renales el efecto de las estatinas sobre la reducción del riesgo cardiovascular y mortalidad global son inciertos, así como sobre la función del injerto ${ }^{10,11,12}$, a pesar de lo cual las guías recomiendan su utilización ${ }^{13}$.

Un aspecto importante con respecto a la seguridad de las estatinas en pacientes trasplantados son sus interacciones, en especial con la ciclosporina y, en menor medida, con el tacrolimus, metabolizados ambos a través del citocromo P450-3A4 $4^{14}$, provocando un aumento de los niveles séricos de estatinas y del riesgo de miopatía. En este sentido, la fluvastatina y la pravastatina son las que ofrecen un menor riesgo de interacciones por su eliminación a través de otras rutas metabólicas, siendo la fluvastatina la que muestra una mejor perfil de seguridad ${ }^{15,16}$. El empleo de otros fármacos con capacidad de interacción sobre el citocromo P450-3A4 deberán ser evitados 0 utilizados con sumo cuidado cuando se administren asociados a estatinas y anticalcineurínicos; éstos incluyen macrólidos (eritromicina, claritromicina), calcioantagonistas (verapamilo, diltiazem) y antifúngicos (fluconazol, itraconazol), entre otros. Aunque rosuvastatina y pitavastatina son metabolizadas por vías diferentes al citocromo P450 su uso concomitante con ciclosporina está contraindicado. Se recomienda evitar la administración de atorvastatina en pacientes tratados con sirolimus por la posible elevación de los niveles séricos de este último, al ser la atorvastatina un potente inhibidor de la glicoproteína transportadora intestinal $(\mathrm{P}-\mathrm{gp})^{17}$. La ciclosporina puede elevar considerablemente los niveles séricos de la ezetimiba, por lo que se recomienda utilizar inicialmente dosis no superiores a $5 \mathrm{mg} / \mathrm{di}^{18}{ }^{18}$.

\section{Tratamiento de la Dislipemia en el Paciente con Infección por el VIH/SIDA}

Los pacientes con infección por VIH tienen un mayor riesgo de desarrollar enfermedades cardiovasculares, no solo relacionado con la influencia de los factores de riesgo tradicionales ${ }^{1,2}$, sino con otros factores: inflamación crónica causada por el VIH, la inmunodeficiencia ${ }^{3}$ y la posible acción directa de ciertos antirretrovirales ${ }^{4}$.

El inicio del tratamiento antirretroviral (TAR) suele provocar una elevación de los lípidos plasmáticos. El incremento del colesterol total y del cLDL es mayor que el del cHDL, lo que

Tabla 4. Dosis recomendadas de estatinas en la ERC, estadios G3-G5 (incluidos pacientes en diálisis y trasplantados)

\begin{tabular}{|l|c|l|}
\hline Estatina & Dosis $(\mathbf{m g})$ & Notas \\
\hline Lovastatina & SD & \\
\hline Fluvastatina & 80 & Reducir dosis $50 \%$ con FGe $<30 \mathrm{ml} / \mathrm{min} / 1,73 \mathrm{~m}^{2}$ \\
\hline Atorvastatina & 20 & \\
\hline Rosuvastatina & 10 & Dosis inicial $5 \mathrm{mg} /$ día con FGe $<30 \mathrm{ml} / \mathrm{min}^{2} 1,73 \mathrm{~m}^{2}$ \\
\hline Simvastatina/ezetimibe & $20 / 10$ & \\
\hline Pravastatina & 40 & Dosis inicial $10 \mathrm{mg}$ con FGe $<60 \mathrm{ml} / \mathrm{min}^{2} 1,73 \mathrm{~m}^{2}$ \\
\hline Simvastatina & 40 & Dosis incicial $5 \mathrm{mg}$ con FGe $<10 \mathrm{ml} / \mathrm{min}_{1} / 1,73 \mathrm{~m}^{2}$ \\
\hline Pitavastatina & 2 & \\
\hline
\end{tabular}

Fuente: KOQI $2003^{3}$ y KDIGO $2013^{5}$ (modificado). SD = sin datos 
Tabla 5. Uso de las estatinas en el paciente con infección por VIH

\begin{tabular}{|l|c|l|c|c|}
\hline Fármaco & Dosis $(\mathbf{m g})$ & Efectos secundarios & TAR con IP/r & TAR con ITINAN \\
\hline Simvastatina & $10-80$ & G-I, Cefalea, Insomnio, Rabdomiolisis, Hepatitis & Contraindicado & Considerar $\uparrow$ dosis \\
\hline Pravastatina & $20-80$ & G-I, Cefalea, Insomnio, Rabdomiolisis, Hepatitis & Considerar $\uparrow$ dosis & Considerar $\uparrow$ dosis \\
\hline Atorvastatina & $10-80$ & G-I, Cefalea, Insomnio, Rabdomiolisis, Hepatitis & Inicio dosis bajas & Considerar $\uparrow$ dosis \\
\hline Fluvastatina & $20-80$ & G-I, Cefalea, Insomnio, Rabdomiolisis, Hepatitis & Considerar $\uparrow$ dosis & Considerar $\uparrow$ dosis \\
\hline Rosuvastatina & $5-40$ & G-I, Cefalea, Insomnio, Rabdomiolisis, Hepatitis & Inicio dosis $<20 \mathrm{mg}$ \\
\hline Pitavastatina & $1-4$ & Mareos, somnolencia, Mialgias & No interacciones \\
\hline
\end{tabular}

se traduce en una ratio mayor del índice aterogénico CT/ HDL. Los inhibidores de proteasa (IP) son los que se han asociado a un mayor número de alteraciones metabólicas ${ }^{4}$, cuya patogénesis es compleja y no completamente conocida. La dislipemia es uno de los efectos secundarios más frecuentes en los pacientes tratados con IP, especialmente cuando están potenciados con ritonavir. Desde el punto de vista analítico se aprecia una elevación del colesterol total, triglicéridos, noHDLc y aumento de la ratio CT/HDL.

Aunque se considera un efecto de clase, el perfil de alteraciones lipídicas varía con el IP utilizado. Es ligeramente mejor en los pacientes tratados con atanazavir, sobre todo cuando no se potencia con ritonavir, que con darunavir o lopinavir.

La evaluación de las ECV en pacientes infectados por el $\mathrm{VIH}$ debe ser esencialmente la misma que se practica en la población general teniendo siempre presente determinados aspectos relacionados con el tratamiento antirretroviral y la importancia del estado inflamatorio persistente asociado cuando la infección viral no está controlada.

Una vez identificados los principales FRCV se procederá a cuantificar el riesgo vascular y a clasificar a los pacientes, según la probabilidad de presentar un evento CV en los siguientes 10 años, como de bajo, medio 0 alto riesgo. Para la cuantificación del riesgo individual se emplean las mismas escalas que en la población general ${ }^{5}$.

Las guías de la Sociedad Europea de Cardiología 6 recomiendan que los pacientes con infección por VIH se consideren al menos de alto riesgo CV y en ellos el objetivo de LDLC debe ser $<100 \mathrm{mg} / \mathrm{dl}$.

El tratamiento de la dislipemia en la infección por VIH no varía sustancialmente al utilizado en la población general. Los fármacos de primera elección en el tratamiento de la hipercolesterolemia son las estatinas ${ }^{5}$, y de ellas, la atorvastatina, debido a su eficacia, seguridad, experiencia y precio, pero también pueden considerarse rosuvastatina y pravastatina. La pitavastatina posee como ventaja principal su escasa capacidad de producir interacciones con el TAR ya que se metaboliza mínimamente a través del citrocromo P450 (CYP) 7,8 . La elección de un fármaco u otro estará basada en la presencia de potenciales interacciones medicamentosas y la intensidad de los trastornos lipídicos (tabla 5). En general se recomienda iniciar tratamiento con dosis bajas e ir incrementando en función de la eficacia y/o toxicidad.

Cuando no se alcanzan los objetivos terapéuticos, más que aumentar las dosis habituales de estatina, que podrían alterar los niveles de los fármacos antirretrovirales 0 aumentar su toxicidad, se podría recurrir a dos estrategias: cambio en la medicación antirretroviral por fármacos que tengan un perfil lipídico menos desfavorable 0 añadir ezetimiba a dosis de $10 \mathrm{mg}$ diarios.

Existen además determinadas combinaciones de TAR que se asocian a un cambio favorable en el perfil lipídico $0^{9,10,11,12}$. Añadir un fármaco hipolipemiante significa añadir complejidad al régimen terapéutico y posibilidad de aparición de nuevos efectos adversos e interacciones. La decisión en este caso debe individualizarse ya que no existen evidencias suficientes que avalen una alternativa.

\section{Tratamiento de la Hiperlipemia en el Paciente Oncológico}

Los pacientes con cáncer que reciben un tratamiento antineoplásico presentan un incremento notable de desarrollar enfermedad cardiovascular. Esto no solo es debido al tratamiento recibido, sino a que el cáncer y la enfermedad cardiovascular comparten con frecuencia factores de riesgo comunes, más allá de la edad.

En el pasado, este riesgo era menos evidente porque la esperanza de vida acortada del paciente no permitía desarrollar complicaciones cardiovasculares, pero la actual eficacia de las terapias lo convierten en un asunto relevante. Además el uso de quimioterápicos, la radioterapia o las terapias moleculares pueden producir daños en el sistema cardiovascular ${ }^{1}$.

La relación entre tratamiento antineoplásico y cardiopatía isquémica, donde se obtiene mayor beneficio en el tratamiento de la dislipemia, es menos frecuente. No obstante, sí que se describe un incremento de incidencia de cardiopatía isquémica en los pacientes sometidos a radioterapia torácica y cervical, con especial atención a las mujeres que 


\begin{tabular}{|l|l|l|}
\hline TRATAMIENTO & NEOPLASIA & EFECTO LIPÍDICO \\
\hline Agonistas de GnRH & Próstata & $\begin{array}{l}\uparrow \text { Colesterol total } \\
\uparrow c-H D L \\
\uparrow c-L D L \\
\uparrow G\end{array}$ \\
\hline Bexacarotene & Linfoma Cutáneo T & $\uparrow$ TG \\
\hline Aspariginasa + Corticoides & Leucemia linfoblástica aguda & $\begin{array}{l}\text { Hipertrigliceridemia grave } \\
\text { (puede precisar plasmaféresis) }\end{array}$ \\
\hline Tamoxifeno & Mama & $\begin{array}{l}\downarrow \text { Colesterol total } \\
\text { c-LDL } \\
\uparrow \text { TG }\end{array}$ \\
\hline Inhibidores de aromatasa (anastrozol) & Mama & $\uparrow c-H D L$ \\
\hline
\end{tabular}

recibieron radioterapia para el cáncer de mama (tasas de eventos coronarios pueden incrementarse $>7 \%$ por cada gray recibido, desde los primeros cinco años tras la radioterapia y hasta las tres décadas posteriores)². Además de los factores de riesgo tradicionales que pueden presentar los pacientes con cáncer, existen peculiaridades de algunos tratamientos antineoplásicos que pueden asociarse a alteraciones del perfil lipídico (se resumen en la tabla posterior) ${ }^{3,4}$. La incidencia de cardiopatía isquémica e ictus está aumentada muchos años después de la exposición a radioterapia para el tratamiento de linfomas y de cáncer de mama ${ }^{5}$ y también del cáncer de cabeza y cuello ${ }^{6}$. Las lesiones vasculares de estos pacientes reúnen las características típicas de la ateroesclerosis: acúmulo lipídico, inflamación y trombosis. La estratificación del riesgo no está bien definida para estos pacientes, pero una aproximación razonable sería considerarlos como sujetos de al menos alto riesgo y marcar objetivos lipídicos y estrategias terapéuticas acordes con su categoría.

No existen recomendaciones específicas sobre la elección de fármacos hipolipemiantes. Las estatinas, al igual que en la población general, son los fármacos de primera elección. La ezetimiba o los secuestradores de ácidos biliares pueden estar indicados cuando las estatinas no sean toleradas o sea preciso el tratamiento combinado.

La controversia inicial sobre un potencial riesgo de desarrollar ciertas neoplasias en el tratamiento prolongado con ezetimiba ha quedado descartada?

Un capítulo específico a tener en cuenta es el de las interacciones farmacológicas de las estatinas con diferentes fármacos antineoplásicos, entre las cuales estarían sobre todo los inhibidores de tirosina-cinasas y, especialmente, dasatinib, erlotinib, imatinib, lapatinib y pazopanib: en el caso de estos fármacos se evitará el uso conjunto con fármacos substratos de P450-3A4, como la lovastatina, atorvastatina y simvastatina, para la cual están descritos elevaciones de los niveles plasmáticos en su uso conjunto. Rosuvastatina, pitavastatina, fluvastatina podrían tener menor riesgo por su mínimo o nulo paso por el P450-3A48.

\section{Dislipemias Familiares e Hipolipemiantes}

Las dislipemias familiares son un conjunto de trastornos hereditarios del metabolismo de las lipoproteínas caracterizadas por alteraciones cuantitativas y/o cualitativas de los lípidos circulantes, y engloban trastornos como Hiperquilomicronemia Familiar, Disbetalipoproteinemia, Hipertrigliceridemia Familiar Endógena, Hiperlipemia Familiar Combinada (HFC) e Hipercolesterolemia Familiar (HF). Por razones de relevancia epidemiológica y clínica, nos centraremos en exponer los objetivos terapéuticos y uso de hipolipemiantes en las dos últimas.

Dependiendo de la definición y la población seleccionada, aproximadamente un $50 \%$ de la población española padece algún tipo de trastorno lipídico'. Sin embargo, no hay estudios que hayan analizado la prevalencia de dislipemias familiares -tampoco a nivel internacional-. Se estima un 0,2\% para HF heterocigota ${ }^{2}$ y $0,5-2 \%$ para $\mathrm{HFC}^{3}$. No deben por tanto considerarse enfermedades raras puesto que supondrían más de 50.000 casos en Galicia con datos del último padrón poblacional (2014).

Su historia natural está ligada al desarrollo de ECV, a menudo precoz, y por ello son considerados procesos de alto $\mathrm{RCV}^{4}$ en los que no se deben utilizar las escalas de valoración habituales (SCORE, Framingham, etc.). Quedan habitualmente excluidos de las recomendaciones de guías de práctica clínica "generales ${ }^{\prime \prime 5,6}$ y se han editado otras guías más específicas ${ }^{7-10}$.

\section{Hiperlipemia Familiar Combinada (HFC)}

Trastorno autosómico dominante de elevada penetrancia pero bases genéticas heterogéneas y poco aclaradas (polimorfismos usf1s2, gen LPL, clusterApo Al-CIII-AIV-AV). La patogenia es compleja pero se sabe que subyace la insulinorresistencia, incluso en no obesos, con hiperaflujo de ácidos grasos libres al hígado y aumento de la síntesis hepática de Apo B y partículas VLDL, cuyo metabolismo rinde LDL pequeñas y densas altamente aterogénicas (fenotipo B). El trastorno se asocia a hipertensión arterial, intolerancia a la glucosa y síndrome metabólico. La dislipemia aparece a partir de la pubertad, y es característica la variabilidad fenotípica inter e intraindividual 
(aumento colesterol LDL y/o triglicéridos), como respuesta a factores exógenos (dieta, alcohol, fármacos) 0 cambios ponderales. Los niveles de CHDL son a menudo bajos y elevados los de Apo B (>125 mg/dL). El trastorno subyace con frecuencia en sujetos con ECV prematura ${ }^{11}$. Además hay riesgo de pancreatitis en quienes presentan hipertrigliceridemia severa (>1.000 mg/dL). Debido a su heterogeneidad no hay unanimidad con los criterios diagnósticos. Los más aceptados en nuestro medio son los propuestos por la Red Temática de Investigación Cardiovascular en Hiperlipidemias Genéticas en 2003 (ISC III), refrendados en un documento de consenso recientemente publicado ${ }^{10}$.

\section{Criterios diagnósticos de la hiperlipidemia familiar combinada}

\section{Persona afectada}

En adultos: colesterol total $>240 \mathrm{mg} / \mathrm{dl}(0 \mathrm{c}-\mathrm{LDL}>160 \mathrm{mg} / \mathrm{dl})$

y/o triglicéridos $>200 \mathrm{mg} / \mathrm{dl}$

En menores de 20 años: colesterol total $>200 \mathrm{mg} / \mathrm{dl}$ (o cLDL >130 mg/dl) y/o triglicéridos > $120 \mathrm{mg} / \mathrm{dl}$

Descartar causas secundarias: índice de masa corporal $>35 \mathrm{~kg} / \mathrm{m}^{2}, \mathrm{HbA} 1 \mathrm{c}>10 \%$, hipotiroidismo no controlado y/o etilismo (> $40 \mathrm{~g}$ alcohol/día)

\section{Familia afectada}

Dos o más miembros de primer grado (padres, hermanos, hijos) afectados de hiperlipidemia mixta, 0 de combinaciones de fenotipos, entre hipercolesterolemia pura (lla), hiperlipidemia mixta (llb) 0 hipertrigliceridemia (IV)

Se excluyen familias con xantomas tendinosos y/o cifras de c-LDL $>300 \mathrm{mg} / \mathrm{dl}$ en 2 o más familiares de primer grado con fenotipo lla Historia familiar de enfermedad cardiovascular ateroesclerótica prematura $(<60$ años)

La historia familiar de ECV prematura no es un criterio estricto de HFC.

El tratamiento de la dislipemia dependerá del fenotipo existente pero el objetivo terapéutico primario debe ser el control del $C L D L$, con recomendaciones basadas en evidencias indirectas:

- En general se aconseja un objetivo de c-LDL $<100 \mathrm{mg} / \mathrm{dL}^{5,10}$.

- En jóvenes (<40 años) sin otros factores de riesgo cardiovascular puede considerarse un objetivo de $\mathrm{CLDL}<130 \mathrm{mg} / \mathrm{dL}^{10}$.

- En pacientes con ECV establecida, ERC moderada/grave 0 diabetes con lesión de órgano diana u otro factor de riesgo añadido, se aconseja un $\mathrm{CLDL}<70 \mathrm{mg} / \mathrm{dl}$, o una reducción mayor al $50 \%$ de los niveles iniciales, cuando no se pueda alcanzar tal objetivo $0^{5,10}$.

Cuando hay hipertrigliceridemia $>400 \mathrm{mg} / \mathrm{dL}$ el cálculo del CLDL mediante la fórmula de Friedewald no es válido, por lo que se cuantificará el CLDL de forma directa 0 se utilizará como objetivo el noHDLc, cuyos niveles objetivo estarán 30 $\mathrm{mg} / \mathrm{dL}$ por encima del objetivo cLDL. Por ejemplo, donde el objetivo cLDL era $<100 \mathrm{mg} / \mathrm{dL}$, el noHDLc deberá ser $<130$ $\mathrm{mg} / \mathrm{dL}$, y así en los demás supuestos.

Para alcanzar el objetivo de CLDL (o noHDLC) el pilar fundamental del tratamiento son la estatinas. Se seleccionará una u otra, así como su dosis, en función de su eficacia, condi- cionantes clínicos del paciente, tolerabilidad y precio. De no alcanzar el objetivo terapéutico con estatinas puede asociarse ezetimiba (10 mg/día) o incluso secuestradores de ácidos biliares (resincolestiramina, colestipol, colesevelam) aunque estos últimos deben de ser considerados como fármacos de tercera línea porque pueden aumentar la trigliceridemia. En intolerantes a estatinas se recomienda usar ezetimiba, asociada o no a otros hipolipemiantes.

Teniendo en cuenta los beneficios observados en pacientes con dislipemia aterogénica (CHDL $<34$ y triglicéridos $>204$ $\mathrm{mg} / \mathrm{dL})^{12}$, en sujetos con HFC que muestren este perfil lipídico y una vez alcanzado el objetivo de cLDL, puede ser útil la adición de fenofibrato al régimen hipolipemiante.

En hipertrigliceridemia severa (>1.000 mg/dL) hay riesgo de pancreatitis ${ }^{13}$ por lo que, además de tener en cuenta el objetivo de noHDLc, se recomienda administrar fibratos u omega-3 (4 gr/día). En este sentido cabe recordar que no se debe asociar gemfibrocilo a estatinas, por el riesgo de rabdomiolisis.

\section{Hipercolesterolemia Familiar (HF)}

Entendemos por HF "monogénica" un conjunto de trastornos del metabolismo lipídico de carácter hereditario, caracterizados por un aumento de 2-3 veces los valores normales de CLDL (190 y 400mg/dL). Aparecen desde edades tempranas de la vida -trigliceridemia generalmente normal- y tienen una elevada incidencia de ECV aterosclerótica, a menudo precoz, con la cardiopatía isquémica como principal causa de muerte. Son patognomónicos los xantomas (tendinosos y cutáneos) y pueden aparecer xantelasmas y arco corneal. Para todo ello deben utilizarse estatinas, en los mismos términos que los expuestos para HFC. Igualmente, cuando no se consigue alcanzar el objetivo de cLDL puede asociarse ezetimiba y/o secuestradores de ácidos biliares, recordando que el efecto de los tres fármacos es aditivo. En intolerantes a estatinas se recomienda el uso de ezetimiba asociada o no a secuestradores de ácidos biliares.

Aunque el diagnóstico definitivo es genético, los criterios de diagnóstico clínico más difundidos son los del Med-Ped holandés ${ }^{14}$ que muestran para un puntuación de 8 puntos una sensibilidad del $41 \%$ y especificidad de $88 \%$.

\section{Criterios Med-Ped para diagnóstico de hipercolesterolemia familiar (tomado de ref. 9).}

Aunque consideramos la HF como de alto riesgo cardiovascular, la presencia de otros FRCV condiciona el riesgo (varones $>30$ años, mujeres $>40$ años; tabaquismo; familiares de primer grado con ECV prematura; CHDL $<40$ mg/dL o Lpa $>50$ mg/ $\mathrm{dL}$ ), así como la existencia de aterosclerosis subclínica 0 ECV, y por tanto también se condiciona el nivel de objetivo terapéutico primario recomendado para cLDL. No se han desarrollado ensayos clínicos específicos en población con HF que demuestren reducción de la ECV con el uso de hipolipemiantes, pero registros de cohortes europeas con HF sí han mostrado que el uso de estatinas se asocia con una mejora del pronóstico cardiovascular, sin modificar la 


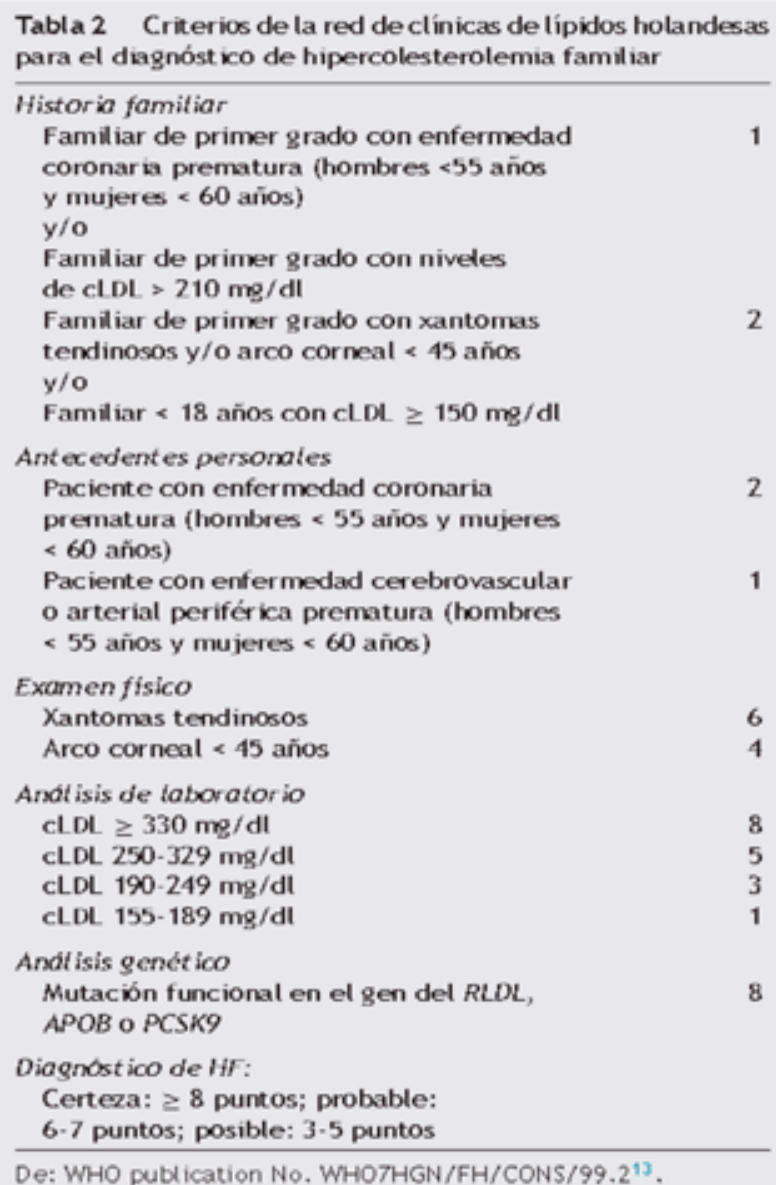

mortalidad no cardiovascular ${ }^{15,16}$.

Los objetivos de control del CLDL, recomendados son los siguientes ${ }^{8,9}$ :

- En pacientes con ECV establecida, diabetes 0 aterosclerosis subclínica se recomienda un c-LDL $<70 \mathrm{mg} / \mathrm{dl}$.

- En los que tienen $\geq 1$ FRCV adicional se aconseja un $\mathrm{cLDL}<100 \mathrm{mg} / \mathrm{dL}$.

- En el resto parece razonable un $\mathrm{CLDL}<130 \mathrm{mg} / \mathrm{dL}$.

- Cuando no se pueda alcanzar el objetivo se aconseja al menos una reducción del 50\% respecto a los niveles iniciales de CLDL.

\section{Estatinas y otros Hipolipemiantes}

Cuando perseguimos disminuir la morbimortalidad cardiovascular en pacientes con dislipemias, las estatinas constituyen el pilar fundamental del tratamiento, aunque no la única herramienta útil para tal fin. La eficacia en este punto ha sido tan relevante con el uso de estatinas que hasta la fecha, salvo ezetimiba, ningún otro hipolipemiante ha demostrado un claro beneficio adicional.

\section{Estatinas}

Las "estatinas" inducen una reducción de las concentraciones plasmáticas de c-LDL del 20\% al 50-60\% (ver tabla del capítulo "prevención primaria") ${ }^{1}$, distinta para cada estatina aunque en todos los casos dosis-dependiente. El mayor efecto en términos relativos es el obtenido con la mínima dosis de cada estatina e inducen reducciones adicionales de cLDL en aproximadamente un 6\% al duplicar la dosis. Aumentan el c-HDL un 4-10\% y disminuyen los triglicéridos un 10-30\% aunque de forma inconstante y variable. Los grandes ensayos clínicos con estatinas tanto en prevención primaria (pravastatina, lovastatina, atorvastatina y rosuvastatina) como secundaria (fluvastatina pravastatina, simvastatina y atorvastatina) nos han mostrado que esa reducción del CLDL se relaciona de forma lineal y continua con la reducción de la incidencia de ECV, en una relación 2:1 en donde por cada $38 \mathrm{mg} / \mathrm{dL}$ de reducción del c-LDL se conseguía una reducción de ECV en un 21\% ${ }^{1}$. Tal relación es independiente de la edad ${ }^{2}$, sexo $^{3} 0$ nivel de riesgo cardiovascular ${ }^{4}$ de los pacientes, haciéndose extensiva a población diabética ${ }^{5}$ y enfermos renales crónicos ${ }^{6}$, salvo dializados o trasplantados renales. Las estatinas son fármacos seguros, con una frecuencia de efectos adversos globales en torno a un $0,5 \%{ }^{7}$, la mayoría de los cuales son dependientes de dosis y en ocasiones relacionados con interacciones farmacocinéticas. Los efectos adversos serios (hipertransaminasemia -x3- 0 elevación de CPK -x10-) son raros y la rabdomiolisis excepcional (1/10.000 tratados respecto a placebo). En los últimos años se ha alertado sobre el efecto diabetógeno de la estatinas, sobre todo estatinas de alta eficacia a dosis elevadas ${ }^{8}$, riesgo que se ve superado en una relación 1:9 por el beneficio cardiovascular que aportan? .

\section{Ezetimiba}

Actúa bloqueando el receptor intestinal del colesterol e inhibe la absorción de éste. Es un hipolipemiante eficaz, muy bien tolerado y seguro, con una tasa de efectos adversos similar a placebo, sin una mayor incidencia de cáncer $^{10}$. A la dosis de $10 \mathrm{mg}$ diarios consigue reducciones medias de colesterol total en un $13 \%$ y de c-LDL en un $18 \%$ cuando se administra en monoterapia y, descensos medios adicionales de colesterol total en un $16 \%$ y cLDL en un $23 \%$ cuando se administra combinado con una estatina en curso. Puede coadministrarse con estatinas, fibratos o secuestradores de ácidos biliares, con un efecto hipolipemiante aditivo. En pacientes con enfermedad renal crónica la administración conjunta de ezetimiba (10 mg/día) y simvastatina (20 mg/ día) redujo un $17 \%$ el riesgo relativo de evento aterosclerótico mayor ${ }^{12}$. Los resultados del ensayo IMPROVE-IT, en prevención secundaria, demuestran un beneficio adicional mediante ezetimiba al tratamiento con estatinas en términos de reducción de la morbimortalidad cardiovascular ${ }^{13}$.

\section{Secuestradores de ácidos biliares (SAB)}

Comercializados en España el colestipol (5-30 gr/día), colestiramina (4-24 gr/día) y colesevelam (2.5-3.75 gr/día). Secuestran los ácidos biliares de la luz intestinal impidiendo su absorción, lo que ocasiona la caída de su reserva corporal que estimula tanto su propia síntesis hepática como la de receptores $L D L$ que hace disminuir los niveles plasmáticos de CLDL un $15-20 \%$. Pueden elevar los triglicéridos sobre todo si ya hay hipertrigliceridemia. Pueden asociarse a esta- 
tinas, ezetimiba o fibratos, con un efecto hipolipemiante aditivo. En varones sin ECV, resincolestiramina redujo frente a placebo un 19\% la morbimortalidad coronaria (ensayo LRCCPPT) ${ }^{14}$. En prevención secundaria la asociación de colestipol con niacina o lovastatina frente a "tratamiento habitual" redujo también la morbimortalidad coronaria ${ }^{15}$. Todos estos datos han sido refrendados por una revisión sistemática que mostró que los SAB disminuyen la mortalidad cardíaca sin modificar la mortalidad por causa no cardiovascular ${ }^{15}$. Se desconoce, sin embargo, su beneficio adicional en pacientes que reciben un tratamiento óptimo con estatinas, pues no se han realizado estudios al respecto. Son fármacos seguros, sin efecto tóxico sistémico, pero limitan la absorción de vitaminas liposolubles y pueden disminuir la biodisponibilidad de otro fármacos (digoxina, propanolol, warfarina, valpróico, tiazidas, estrógenos y progestágenos) si se administran de forma conjunta. Tienen problemas de palatabilidad y son constipantes por lo que tienen una frecuencia de abandono de un 40-60\% en los ensayos clínicos.

\section{Fibratos}

Gemfibrozilo, fenofibrato y bezafibrato. Activan los receptores PPAR $\alpha$ y regulan la expresión de genes implicados en el metabolismo lipídico. Disminuyen la producción hepática de VLDL, potencian el efecto de la lipoproteinlipasa y lipasa hepática y disminuyen la expresión de APO Clll. Con ello, reducen la trigliceridemia entre un $20-50 \%$ (efecto principal), pero apenas bajan el cLDL (5-10\%) 0 incluso lo pueden aumentar a la vez que aumentan ligeramente el cHDL (5$15 \%)$. En la era "pre-estatinas" gemfibrozilo en monoterapia, disminuyó la incidencia de ECV un 34\% en prevención primaria ${ }^{16}$ y un $22 \%$ en prevención secundaria ${ }^{17}$, beneficios que no se lograron de forma estadísticamente significativa con otros fibratos. Tampoco ha sido demostrado beneficio adicional al tratamiento con estatinas sobre la reducción de ECV. Una revisión sistemática y metaanálisis que incluyó 18 ensayos clínicos (45.000 pacientes) mostraba que los fibratos reducen en un 10\% la morbimortalidad cardiovascular sin modificar la mortalidad general ${ }^{18}$. Pueden provocar litiasis biliar, hipertransaminasemia ligera y transitoria e incluso deterioro de función renal. Mención aparte merece el riesgo de miotoxicidad, incluso rabdomiolisis, especialmente en presencia de hipotiroidismo, insuficiencia renal o edad avanzada, y tras interacciones farmacológicas, particularmente en el tratamiento conjunto con estatinas. Por ello no se recomienda asociar gemfibrozilo con estatinas.

\section{Acído nicotínico}

Ha dejado de comercializarse en nuestro país.

\section{Acidos grasos omega 3}

Incluyen el acido eicosapentanoico (EPA) y docosahexanoico (DHA). A dosis no hipolipemante de 1,7 g/día demostraron reducir la morbimortalidad cardiovascular, sobre todo la muerte súbita ${ }^{19}$. A dosis mayores $(4 \mathrm{~g} / \mathrm{día}$ ) reduciendo los triglicéridos aproximadamente un $30 \%$, con un ligero efecto sobre cHDL (aumento 3\%) y sin efecto o mínimo aumento de c-LDL. Asociando $1.800 \mathrm{mg}$ de EPA con estatinas en un ensayo abierto frente a placebo, se consiguió reducir la incidencia de $\mathrm{ECV}^{20}$ pero los intentos por demostrar un beneficio cardiovascular adicional en pacientes ya tratados con estatinas han fracasado $0^{21,22}$.

\section{Bibliografía}

\section{Introducción}

1. World Health Organization. Global status report on noncommunicable disaeses 2010. Geneva: WHO; 2011

2. Organización Mundial de la Salud [Internet]. Geneva: World Health Organization (CH); [consultado el 18 Julio 2014]. Disponible en: http://www.who.int/mediacentre/factsheets/fs310/es/index2.html

3. Bloom DE, Cafiero E, Jané-Llopis E, Abrahams-Gessel S, Bloom L, Fathima S, et al. The Global Economic Burden of Noncommunicable Diseases. 2011. Geneva: World Economic Forum

4. Perk J, De Backer G, Gohlke H, Graham I, Reiner Z, Verschuren M, et al. European Guidelines on cardiovascular disease prevention in clinical practice (version 2012). Eur J Prev Cardiol 2012;19:585-667

5. Perk J, De Backer G, Gohlke H, Graham I, Reiner Z, Verschuren M, et al. Guía europea sobre prevención de la enfermedad cardiovascular en la práctica clínica (versión 2012). Rev Esp Cardiol. 2012;65:e1-e66

6. Cohen M, Diez J, Levine G, Ferguson J, Morrow D, Rao S, et al. Pharmacoinvasive management of acute coronary syndrome: incorporating the 2007 ACC/AHA guidelines: the CATH (cardiac catheterization and antithrombotic therapy in thehospital) Clinical Consensus Panel Report-III. J Invasive Cardiol.2007;19:525-538

7. Ministerio de Sanidad Servicios Sociales e Igualdad. Informe de utilización de medicamentos. Utilización de medicamentos hipolipemiantes en España durante el periodo 2000-2012. Madrid: MSSSI; 2014

8. Reiner Z, Catapano A, De Backer G, Graham I, Taskinen M, Wiklund O, et al. ESC/ EAS guidelines for the management of dyslipideamias. The Task Force for the management of dyslipidaemias of the European Society of Cardiology (ESC) and the European Atherosclerosis Society (EAS). Eur Heart J 2011;32:1769-1878.

9. Perk J, De Backer G, Gohlke H, Graham I, Reiner Z, Verschuren M et al. European Guidelines in cardiovascular disease prevention in clinical practice (version 2012). The Fith Joint Task Force of The European Society of Cardiology and Other Societies on Cardiovascular Disease Prevention in clinical Practice. Eur Heart J doi: 10.1093/eurheart//ehs092.

10. Goff D, Lloyd-Jones D, Bennett G, Coady S, D’Agostino R, Gibbons R et al. 2013 ACC/AHA guideline on the assessment of cardiovascular risk: a report of the American College of Cardiology/American Heart Association Task Force on Practice Guidelines. Circulation. 2014;129(25 Suppl 2):S74-S75

11. Ioannidis J. More than a billion people taking statins? Potential implications of the new cardiovascular guidelines. JAMA. 2014;311:463-464

12. National Institute for Health and Care Excellence. Lipid modification: cardiovascular Risk assessment and the modification Of blood lipids for the primary and Secondary prevention of Cardiovascular disease Issued. London: NICE; 2014. Disponible en: http://www.nice.org.uk/news/article/wider-use-of-statins-could-cut-deaths-fromheart-disease. [consultado el 18 Julio 2014]

13. National Cholesterol Education Program (NCEP) Expert Panel on Detection, Evaluation, and Treatment of High Blood Cholesterol in Adults (Adult Treatment Panel III). Third Report of the National Cholesterol Education Program (NCEP) Expert Panel on Detection, Evaluation, and Treatment of High Blood Cholesterol in Adults (Adult Treatment Panel III) final report. Circulation. 2002;106:3143-3421

14. Ministerio de Sanidad y Consumo. Estudio APEAS. Estudio sobre la seguridad de los pacientes en Atención Primaria de Salud. Madrid: MSC; 2008

Definición de objetivos de tratamiento

1. Perk J, De Backer G, Gohlke H, Graham I, Reiner Z, Verschuren M, et al. European Guidelines on cardiovascular disease prevention in clinical practice (version 2012). Eur J Prev Cardiol 2012;19:585-667

2. Reiner Z, Catapano A, De Backer G, Graham I, Taskinen M, Wiklund O, et al. ESC/ EAS guidelines for the management of dyslipideamias. The Task Force for the management of dyslipidaemias of the European Society of Cardiology (ESC) and the European Atherosclerosis Society (EAS). Eur Heart J 2011;32:1769-1878

3. Goff D, Lloyd-Jones D, Bennett G, Coady S, D’Agostino R, Gibbons R et al. 2013 ACC/AHA guideline on the assessment of cardiovascular risk: a report of the American College of Cardiology/American Heart Association Task Force on Practice Guidelines. Circulation. 2014;129(25 Suppl 2):S74-S75

4. National Institute for Health and Care Excellence. Lipid modification: cardiovascular Risk assessment and the modification Of blood lipids for the primary and Secondary prevention of Cardiovascular disease Issued. London: NICE;2014

5. National Cholesterol Education Program (NCEP) Expert Panel on Detection, Evaluation, and Treatment of High Blood Cholesterol in Adults (Adult Treatment Panel III). 
Third Report of the National Cholesterol Education Program (NCEP) Expert Panel on Detection, Evaluation, and Treatment of High Blood Cholesterol in Adults (Adult Treatment Panel III) final report. Circulation. 2002;106:3143-421

6. Baigent C, Keech A, Kearney P, Blackwell L, Buck G, Pollicino C, et al. Efficacy and safety of cholesterol-lowering treatment: prospective meta-analysis of data from 90,056 participants in 14 randomised trials of statins. Lancet 2005; 366(9493):1267-1278

7. Baigent C, Blackwell L, Emberson J, Holland L, Reith C, Bhala N, et al. Efficacy and safety of more intensive lowering of LDL cholesterol: a meta-analysis of data from 170,000 participants in 26 randomised trials. Lancet 2010;376:1670-1681

8. Reiner Z, Catapano A, De Backer G, Graham I, Taskinen M, Wiklund O, et al. Guía de la ESC/EAS sobre el manejo de las dislipemias. Rev Esp Cardiol. 2011;64:1168.e1-e60

\section{Prevención Primaria}

1. Taylor F, Huffman M, Macedo A, Moore T, Burke M, Davey Smith G, et al. Statins for the primary prevention of cardiovascular disease. Cochrane Database Syst Rev. 2013;1:CD00481

2. Mihaylova B, Emberson J, Blackwell L, Keech A, Simes J, Barnes EH, et al. Cholesterol Treatment Trialists' (CT) Collaboration. The effects of lowering LDL cholesterol with statin therapy in people atlow risk of vascular disease: meta-analysis of individual data from27 randomised trials. Lancet. 2012;380(9841):581-90

4. Stone J, Robinson J, Lichtenstein A, Bairey-Merz C, Blum C, Eckel R, et al. 2013 ACC/AHA Guideline on the Treatment of Blood Cholesterol to Reduce Atherosclerotic Cardiovascular Risk in Adults: A Report of the American College of Cardiology/ American Heart Association Task Force on Practice Guidelines. Circulation. 2013;01. cir.0000437738.63853.7a

5. Rabar S, Harker M, O'Flynn N, Wierzbicki AS, Guideline Development Group. Lipid modification and cardiovascular risk assessment for the primary and secondary prevention of cardiovascular disease: summary of updated NICE guidance. BMJ 2014;349:g4356

\section{Prevención secundaria en cardiopatía isquémica}

\section{y arteriopatía periférica}

1. Baigent C, Keech A, Kearney PM, Blackwell L, Buck G, Pollicino C, et al. Efficacy and safety of cholesterol-lowering treatment: prospective meta-analysis of data from 90,056 participants in 14 randomised trials of statins. Lancet. 2005;366:1267-78

2. Bezafibrate Infarction Preventions. Secondary prevention by raising HDL cholesterol and reducing triglycerides in patients with coronary artery disease. Circulation. 2000;102:21-27

3. Efficacy and safety of more intensive lowering of LDL cholesterol: a meta-analysis of data from 170000 participants in 26 randomised trials. Cholesterol Treatment Trialists (CTT) Collaboration. Lancet, 376:1670-1681

4. Reiner Z, Catapano A, De Backer G, Graham I, Taskinen M, Wiklund O, et al. ESC/EAS guidelines for the management of dyslipideamias. The Task Force for the management of dyslipidaemias of the European Society of Cardiology (ESC) and the European Atherosclerosis Society (EAS). Eur Heart J 2011;32:1769-1878

5. Cannon C, Blazing M, Giugliano A, McCagg A, White J, Theroux P, et al. Ezetimibe added to statin therapy after acute coronary syndromes. N Engl J Med 2015;375:2387-2397

\section{Prevención secundaria en ictus isquémico}

1. Crouse J, Byington R, Hoen H, Furberg C. Reductase inhibitor monotherapy and stroke prevention. Arch Intern Med 1997;157:1305.

2. Piechowski-Jóžwiak B, Bogousslavsky J. Cholesterol as a risk factor for stroke: the fugitive? Stroke 2004; 35:1523.

3. Barter P, Gotto A, LaRosa J, Maroni J, Szarek M, Grundy SM, et al. for the Treating to New Targets Investigators. HDL cholesterol, very low levels of LDL cholesterol, and cardiovascular events. N Engl J Med. 2007;357:1301-1310

4. Bucher $\mathrm{HC}$, Griffith LE, Guyatt GH. Effect of HMGcoA reductase inhibitors on stroke. A meta-analysis of randomized, controlled trials. Ann Intern Med 1998; $128: 89$.

5. The AIM-HIGH Investigators. Boden W, Probstfield J, Anderson T, Chaitman B, Desvignes-Nickens P, Koprowicz K, et al. Niacin in patients with low HDL cholesterol levels receiving intensive statin therapy. N Engl J Med. 2011;365:2255-2267

6. Crouse J, Byington R, Bond M, Espeland M, Sprinkle J, McGovern M, et al. Pravastatin, lipids, and atherosclerosis in the carotid arteries: design features of a clinical trial with carotid atherosclerosis outcome. Control Clin Trials 1992;13:495-506

7. Pahan K, Sheikh F, Namboodiri A, Singh I. Lovastatin and ophenylacetate inhibit the induction of nitric oxide synthase and cytokines in rat primary astrocytes, microglia and macrophages. J Clin Invest. 1997;100:2671-9

8. Chen J, Zhang ZG, Li Y, Wang Y, Wang L, Jiang H, et al. Statins induce angiogenesis, neurogenesis, and synaptogenesis after stroke. Ann Neurol. 2003:53:743-51

9. Flint $A$, Kamel $H$, Navi B, Rao V, Faigeles B, Conell $C$, et al. Statin use during ischemic stroke hospitalization is strongly associated with improved poststroke survival. Stroke. 2012;43:147-54

10. The Stroke Prevention by Aggressive Reduction in Cholesterol Levels (SPARCL) Investigators. High-dose atorvastatin after stroke or transient ischemic attack. N Engl J Med. 2006;355:549-559

11. Huisa B, Stemer A, Zivin J. Atorvastatin in stroke: a review of SPARCL and subgroup analysis. Vascular Health and Risk Management 2010:6 229-236

12. Amarenco P, Goldstein LB, Szarek M, Sillesen H, Rudolph A, Callahan A, et al. Effects of intense low-density lipoprotein cholesterol reduction in patients with stroke or transient schemic attack: the Stroke Prevention by Aggressive Reduction in Cholesterol Levels (SPARCL) trial. Stroke. 2007:38:3198-3204

\section{Uso de hipolipemiantes en ancianos}

1. Strandberg $T$, Kolehmainen $L$, Vuorio $A$. Evaluation and treatment of older patients with hypercholesterolemia. A clinical review. JAMA 2014;312:1136-1144

2. Aronow W. Treatment of high-risk older patients with lipid-lowering therapy. Am J Ther 2008; 15:102-107

3. Thomas JE, Tershakovec AM, Jones-Burton C, Sayeed RA. Foody JM. Lipid lowering for secondary prevention of cardiovascular disease in older adults. Drus Aging 2010;27:959-972

4. Ali R, Alexander K. Statins for the primary prevention of cardiovascular events in older adults: a review of the evidence. Am J Geriatr Pharmacother 2007;5:52-63

5. Gomez-Huelgas R, Martínez-Sellés M, Formiga F, Alemán J, Camafort M, Galve E, et al. Tratamiento de los factores de riesgo cardiovascular en el paciente mayor de 80 años. Med Clin 2014;143:134.e1-134.e11

6. Savarese G, Gotto A, Paolillo S, D’Amore C, Losco T, Musella F, et al. Benefits of statin in elderly subjects without established cardiovascular disease. J Am Coll Cardiol 2013;62:2090-2099

7. Chokshi N, Messerli F, Sutin D, Supariwata A, Shah N. Appropriateness of Stains in patients $\geq 80$ years and comparison to other age groups. Am J Cardiol 2012;110:14771481

8. Hilmer S, Gnjidic D. Statins in older adults. Aust Prescr 2013;36:79-82

9. Clement KM, Walker SW. Statins and their interactions with other lipid-modidying medications. Ther Adv in Drug Safe 2012;3:35-46

10. Reiner Z, Catapano A, De Backer G, Graham I, Taskinen M, Wiklund O, et al. ESC/EAS guidelines for the management of dyslipideamias. The Task Force for the management of dyslipidaemias of the European Society of Cardiology (ESC) and the European Atherosclerosis Society (EAS). Eur Heart J 2011;32:1769-1878

11. Reiner Z. Primary prevention of cardiovascular disease with statins in the elderly. Curr Atheroscler Rep 2014;16:420

12. Stone NJ, Intwala S. Statins in very elderly adults (debate). J Am Geriatr Soc 2014;62:943-945

13. Stone N, Robinson J, Lichtenstein A, Bairey C, Blum C, Eckrl R et al. ACC/AHA Guideline on the Treatment of Blood Cholesterol to Reduce Atherosclerotic Cardiovascular Risk in Adults: a report of the American College of Cardiology/American Heart Association Task Force on Practice Guidelines. Circulation. doi:10.1161/01.cir.0000437738.63853.7a

14. Cannon C, Blazing M, Giugliano A, McCagg A, White J, Theroux P, et al. Ezetimibe added to statin therapy after acute coronary syndromes. N Engl J Med 2015;375:2387-2397

\section{Uso de hipolipemiantes en diabetes}

1. Pedro-Botet $J$, Benaiges $D$, Pedragosa $A$. Dislipemia diabética, macro y microangiopatía. Clin Invest Arterioscl 2012;24:299-305

2. Shah $A D$, Langenberg $C$, Rapsomaniki E, Denaxas S, Pujades-Rodriguez M, Gale $C$ et al. Type 2 diabetes and incidence of cardiovascular diseases: a cohort study in 1.9 million people. Lancet Diabetes Endocrinol 2015;3:105-113

3. Wu L, Parhofer K. Diabetic dyslipidemia. Metabolism 2014;63:1469-1479

4. Shah S, Casas J, Drenos F, Whittaker J, Deanfield J, Swerdlow D, et al. Causal relevance of blood lipid fractions in the development of carotid atherosclerosis: Mendelian randomization analysis. Circ Cardiovasc Genet 2013;6:63-72

5. Do R, Willer C, Schmidt E, Sengupta S, Gao C, Peloso G, et al. Common variants associated with plasma triglycerides and risk of coronary artery disease. Nat Genet 2013:45:1345-1352

6. Howard B, Robbins D, Sievers M, Lee E, Rhoades D, Devereux R, et al. LDL cholesterol as a strong predictor of coronary heart disease in diabetic individuals with insulin resistance and low LDL: the Strong Heart Study. Arterioscl Thromb Vasc Biol 2000;20:830-835

7. Rydén L, Grant P, Anker S, Berne C, Cosentino F, Danchin N, et al. ESC Guidelines on diabetes, pre-diabetes, and cardiovascular diseases developed in collaboration with the EASD: The Task Force on diabetes, pre-diabetes, and cardiovascular diseases of the European Society of Cardiology (ESC) and developed in collaboration with the European Association for the Study of Diabetes (EASD). Eur Heart J 2013;34:3035-3087

8. Jiang R, Schulze M, Li T, Rifai N, Stampfer M, Rimm E, et al. Non-HDL and apolipoprotein B predict cardiovascular disease events among men with type 2 diabetes. Diabetes Care 2004:27:1991-1997

9. Cannon C, Blazing M, Giugliano A, McCagg A, White J, Theroux P, et al. Ezetimibe added to statin therapy after acute coronary syndromes. N Engl J Med 2015;375:2387-2397

10. Sattar N, Preiss D, Murray H, Welsh P, Buckley B, de Craen A, et al. Statins and risk of incident diabetes: a collaborative meta-analysis of randomised statin trials. Lancet 2010;375:735-742

11. Grumprech J, Gosho M, Hounslow N. Comparative long-term efficacy and tolerability of pitavastatin $4 \mathrm{mg}$ and atorvastatin $20 \mathrm{mg}$ in patients with type 2 diabetes mellitus and combined (mixed) dyslipidemia. Diab Obes Metab 2011; 13: 1047-1055 
12. Odawara M, Yamazaki T, Kishimoto J, Ito C, Noda M, Terauchi Y, et al. Effect of pitavastatin on the incidence of diabetes in Japanese individuals with impaired glucose tolerance. 49th European Association for the Study of Diabetes Annual Meeting. 2013. http://easdvirtualmeeting.org/resources/3645

\section{Tratamiento de la Dislipemia}

\section{en la Enfermedad Renal Crónica}

1. Tonelli M, Wiebe N, Culleton B, House A, Rabbat C, Fok M, et al. Chronic kidney disease and mortality risk: a systematic review. J Am Soc Nephrol 2006;17:2034-2047

2. Vaziri N. Dyslipidemia of chronic renal failure: the nature, mechanisms, and potential consequences. Am J Physiol Renal Physiol 2006;290:F262-F272

3. Kidney Disease: Improving Global Outcomes (KDIGO) Lipid Work Group. KDIGO clinical practice guideline for lipid managment in chronic kidney disease. Kidney Int 2013:Suppl 3:259-305

4. Hayward $\mathrm{R}$, Krumholz $\mathrm{H}$. Three reasons to abandon low-density lipoprotein targets: an open letter to the Adult Treatment Panel IV of the National Institutes of Health. Circ Cardiovasc Qual Outcomes 2012;5:2-5

5. Palmer S, Navaneethan S, Craig J, Johnson D, Perkovic V, Hegbrant J, et al. HMG $\mathrm{CoA}$ reductase inhibitors (statins) for people with chronic kidney disease not requiring dialysis. Cochrane Database of Systematic Reviews 2014, Issue 5. Art. No.: CD007784. DOI: 10.1002/14651858.CD007784.pub2

6. Baigent C, Landray M, Reith C, Emberson J, Wheeler D, Tomson C, et al. The effects of lowering LDL cholesterol with simvastatin plus ezetimibe in patients with chronic kidney disease (Study of Heart and Renal Protection): a randomised placebo-controlled trial. Lancet 2011;377:2181-2192

7. Sarnak M, Bloom R, Muntner P, Rahman M, Saland J, Wilson P, et al. KDOQI US Commentary on the $2013 \mathrm{KDIGO}$ Clinical Practice Guideline for Lipid Management in CKD. Am J Kidney Dis 2014 Nov 18. pii: S0272-6386(14)01283-9. doi: 10.1053/j. ajkd.2014.10.005

8. Kong X, Yuan H, Fan J, Li Z, Wu T, Jiang L. Lipid-lowering agents for nephrotic syndrome. Cochrane Database of Systematic Reviews 2013, Issue 12. Art. No.: CD005425. DOI: 10.1002/14651858.CD005425.pub2

9. Stone J, Robinson J, Lichtenstein A, Bairey-Merz C, Blum C, Eckel R, et al. 2013 ACC/AHA Guideline on the Treatment of Blood Cholesterol to Reduce Atherosclerotic Cardiovascular Risk in Adults: A Report of the American College of Cardiology/ American Heart Association Task Force on Practice Guidelines. Circulation. 2013;01. cir.0000437738.63853.7a

10. Wanner C, Krane V, März W, Olschewski M, Mann J, Rufet G, etal. Atorvastatin in patients with type 2 diabetes mellitus undergoing hemodialysis. N Engl J Med 2005;353(3):238-248

11. Fellstrom B, Jardine A, Schmieder R, Holdaas H, Bannister K, Beutler J, et al. Rosuvastatin and cardiovascular events in patients undergoing hemodialysis. N Engl J Med 2009;360:1395-1407

12. Palmer S, Navaneethan S, Craig J, Johnson D, Perkovic V, Nigwekar S, et al. HMG CoA reductase inhibitors (statins) for dialysis patients. Cochrane Database of Systematic Reviews 2013, Issue 9. Art. №.: CD004289. D0I: 10.1002/14651858.CD004289.pub5

13. Holdaas H, Fellstrom B, Jardine A, Holme I, Nyberg G, Fauchald P, et al. Effect of fluvastatin on cardiac outcomes in renal transplant recipients: a multicentre, randomised, placebo-controlled trial. Lancet 2003:361:2024-2031

14. Palmer S, Navaneethan S, Craig J, Perkovic V, Johnson D, Nigwekar S, et al. HMG CoA reductase inhibitors (statins) for kidney transplant recipients. Cochrane Database of Systematic Reviews 2014, Issue 1. Art. No.: CD005019. D0I: 10.1002/14651858. CD005019.pub4

15. Harper CR, Jacobson TA. Managing dyslipidemia in chronic kidney disease. J Am Coll Cardiol 2008:51:2375-2384

16. Reiner Z, Catapano A, De Backer G, Graham I, Taskinen M, Wiklund O, et al. ESC/ EAS guidelines for the management of dyslipideamias. The Task Force for the management of dyslipidaemias of the European Society of Cardiology (ESC) and the European Atherosclerosis Society (EAS). Eur Heart J 2011;32:1769-1878

\section{Tratamiento de la Dislipemia en el Paciente Trasplantado}

1. Massy Z. Hyperlipidemia and cardiovascular disease after organ transplantation. Transplantation 2001;72(Suppl 6):S13-S15

2. Cofan F, Alonso-Melgar A, Canal C, Crespo M, Errasti P, Fijo J, et al. Inmunosupresores y alteraciones de los lípidos. Nefrologia Sup Ext 2013;4:35-40

3. Kobashigawa J, Katznelson S, Laks H, Johnson J, Yeatman L, Wang X, et al. Effect of pravastatin on outcomes after cardiac transplantation. N Engl J Med 1995;333:621-627

4. Weenie K, Meiser B, Thiery J, Nagel D, von Scheidt W, Steinbeck G,et al. Simvastatin reduces graft vessel disease and mortality after heart transplantation: a four-year randomized trial. Circulation 1997;96:1398-1402

5. Kobashigawa J, Moriguchi J, Laks H, Wener L, Hage A, Hamilton M, et al. Ten-Year Follow-up of a Randomized Trial of Pravastatin in Heart Transplant Patients. J Heart Lung Transplant 2005;24:1736-1740

6. Costanzo M, Dipchand A, Starling R, Anderson A, Chan M, Desai S, et al. The International Society of Heart and Lung Transplantation Guidelines for the care of heart transplant recipients. J Heart and Lung Transplant 2010;29:914-956
7. Kobashigawa J. Statins in solid organ transplantation: is there an immunosuppressive effect? Am J Transplant 2004:4:1013-1018

8. Wu A, Ballantyne C, Short B, Torre-Amione G, Young J, Ventura H, et al. Statin use and risks of death or fatal rejection in the Heart Transplant Lipid Registry. Am J Cardiol 2005; 95: 367-72

9. Fröhlich G, Rufibach K, Enseleit F, Wolfrum M, von Babo M, Frank M, et al. Statins and the risk of cancer after heart transplantation. Circulation 2012:126:440-447

10. Holdaas H, Fellstrom B, Jardine A, Holme I, Nyberg G, Fauchald P, et al. Effect of fluvastatin on cardiac outcomes in renal transplant recipients: a multicentre, randomised, placebo-controlled trial. Lancet 2003;361:2024-2031

11. Palmer S, Navaneethan S, Craig J, Perkovic V, Johnson D, Nigwekar S, et al. HMG CoA reductase inhibitors (statins) for kidney transplant recipients. Cochrane Database of Systematic Reviews 2014, Issue 1. Art. No.: CD005019. DOI: 10.1002/14651858. CD005019.pub4

12. Younas N, Wu C, Shapiro R, McCauley J, Johnston J, Tan H, et al. HMG-CoA reductase inhibitors in kidney transplant recipients receiving tacrolimus: statins not associated with improved patient or graft survival. BMC Nephrology 2010;11:5-14

13. Kidney Disease: Improving Global Outcomes (KDIGO) Lipid Work Group. KDIGO clinical practice guideline for lipid management in chronic kidney disease. Kidney Int 2013;Suppl 3:259-305

14. Amundsen R, Åsberg A, Ohm K, Christensen H. Cyclosporine A- and tacrolimus-mediated inhibition of CYP3A4 and CYP3A5 in vitro. Drug Metab Dispos 2012;40:655-661

15. Corsini A, Bellosta S, Baetta R, Fumagalli R, Paoletti R, Bernini F. New insights into the pharmacodynamic and pharmacokinetic properties of statins. Pharmacol Ther 1999;84:413-428

16. Holdaa H, Julian D. The use of statins after solid organ transplantation. Nephrol Dial Transplant 2002;17:1537

17. Wessler J, Grip L, Mendell J, Giugliano R. The P-Glycoprotein transport system and cardiovascular drugs. J Am Coll Cardiol 2013;61:2495-2502

18. Koshman S, Lalonde L, Burton I, Tymchak W, Pearson G. Supratherapeutic response to ezetimibe administered with cyclosporine. Ann Pharmacother 2005:39:1561-1565

\section{Tratamiento de la Dislipemia en}

\section{el Paciente con Infección por el VIH/SIDA}

1. Masia M, Gutierrez F. HIV-related cardiovascular risk factors. Enferm Infecc Microbiol Clin. 2009 Suppl 1:17-23.

2. Obel N, Thomsen H, Kronborg G, Larsen C, Hildebrandt P, Sorensen H, et al. Ischemic heart disease in HIV-infected and HIV-uninfected individuals: a population-based cohort study. Clin Infect Dis. 2007;44:1625-1631.

3. Lichtenstein K, Armon C, Buchacz K, Chmiel J, Buckner K, Tedaldi E, et al. Low CD4+ T cell count is a risk factor for cardiovascular disease events in the HIV outpatient study. Clin Infect Dis. 2010;51:435-447.

4. Mary-Krause M, Cotte L, Simon A, Partisani M, Costagliola D. Increased risk of myocardial infarction with duration of protease inhibitor therapy in HIV-infected men. AIDS. 2003;17:2479-86

5. Grupo de Expertos del grupo de estudio sobre Alteraciones metabólicas (GEAM), de la Secretaría del Plan nacional sobre el SIDA (SPNS) y del Grupo de estudio de SIDA (GeSIDA). Documento de consenso sobre las alteraciones metabólicas y riesgo cardiovascular en pacientes con infección por el virus de inmunodeficiencia humana. Enferm Infecc Microbiol Clin. 2015;33:40.e1-40.e16

6. Reiner Z, Catapano A, De Backer G, Graham I, Taskinen M, Wiklund O, et al. ESC/ EAS guidelines for the management of dyslipideamias. The Task Force for the management of dyslipidaemias of the European Society of Cardiology (ESC) and the European Atherosclerosis Society (EAS). Eur Heart J 2011;32:1769-1878

7. Morgan R, Campbell S, Suehira K, Sponseller C, Yu C, Medlock M. Efects of steadystate lopinavir-ritonavir on the pharmacokinetics of pitavastatin in healthy adult volunteers. JAIDS 2012;60:158-164

8. Yu C, Campbel S, Sponseller C, Knadler M, Small D, Medlock M, et al. Steady-state pharmacokinetics of Darunavir/Ritonavir with Pitavastatin in healthy adult volunteers. AIDS Conference. July 22-27, 2012, Washington, DC, USA. Abstract nTUPE053

9. Martínez E, Arnaiz JA, Podzamczer D, Dalmau D, Ribera E, Domingo P, et al. Substitution of nevirapine, efavirenz, or abacavir for protease inhibitors in patients with immunodeficiency virus infection. N Engl J Med 2003:349:1036-1046

10. Fisher M, Palella F, Tebas P, Gazzard B, Ruane P, van Lunzen J, et al. SPIRIT: switching to emtricitabine/rilpivirine/tenofovir DF single-tablet regimen from boosted protease inhibitor maintains HIV suppression at week 48. 11th International Congress on Drug Therapy in HIV Infection; November 11-15, 2012; Glasgow, UK. Abstract n P285

11. Martínez E, d'Albuquerque PM, Pérez I, Pich JM, Gatell JM. Abacavir/lamivudine versus tenofovir/emtricitabine in virologically suppressed patients switching from ritonavir-boosted protease inhibitors to raltegravir. AIDS Res Hum Retroviruses 2013;29:235-241.

12. Campo R, DeJesus E, Bredeek UF, Henry K, Khanlou H, Logue K, et al. SWIFT: prospective 48-week study to evaluate efficacy and safety of switching to emtricitabine/tenofovir from lamivudine/abacavir in virologically suppressed HIV-1 infected patients on a boosted protease inhibitor containing antiretroviral regimen. Clin Infect Dis. 2013;56:1637-1645 
Tratamiento de la hiperlipemia en el paciente oncológico

1. Albini A, Pennesi G, Donatelli F, Cammarota R, De Flora S, Noonan D. Cardiotoxicity of anticancer drugs: the need for cardio-oncology and cardio-oncological prevention. J Natl Cancer Inst 2010;102:14-25

2. Darby S, Ewertz M, McGale P, Bennet A, Blom-Goldman U, Bronnum D, et al. Risk of ischemic heart disease in women after radiotherapy for breast cancer. $\mathrm{N}$ Engl J Med 2013;368:987-998

3. Saylor P, Smith M. Metabolic complications of androgen deprivation therapy for prostate cancer. J Urol 2009;181:1998-2006

4. Bielecka-Dabrowa A, Hannam S, Rysz J, Banach M. Malignancy-associated dyslipidemia. Open Cardiovasc Med J 2011;5:35-40

5. Darby S, McGale P, Peto R, Granath F, Hall P, Ekbom A. Mortality from cardiovascular disease more than 10 years after radiotherapy for breast cancer: nationwide cohort study of 90000 Swedish women. BMJ 2003;326:256-7

6. Smith G, Smith B, Buchholz T, Giordano S, Garden A, Woodward W, et al. Cerebrovascular disease risk in older head and neck cancer patients after radiotherapy. J Clin Oncol 2008:26:5119-25

7. Peto R, Emberson J, Landray M, Baigent C, Collins R, Clare R, et al. Analyses of cancer data from three ezetimibe trials. N Engl J Med 2008:359:1357-66

8. Sánchez Gómez E, Arco Prados Y. Revisión de las interacciones farmacológicas de los fármacos antineoplásicos orales dispensados en un servicio de farmacia. Farm Hosp. 2014;38:338-363

\section{Dislipemias Familiares e Hipolipemiantes}

1. Gutiérrez Fuentes J, Gómez Gerique J, Gómez de la Cámara A, Rubio M, García Hernández Arístegui I. Dieta y riesgo cardiovascular en España (DRECE II). Descripción de la evolución del perfil cardiovascular. Med Clin 2000;115:726-729

2. Goldstein J, Hobbs H, Brown M. Familial hypercholesterolaemia. En: Scriver C, Beau$\operatorname{det}$ A, Sly W, Valle D, editors. The metabolic and molecular bases of inherited disease. New York: McGraw Hill 1995;p.1981-2030

3. Hazzard W, Goldstein J, Schrott H, Motulsky A, Bierman E. Hyperlipidemia in Coronary Heart Disease III. Evaluation of lipoprotein phenotypes of 156 genetically defined survivors of myocardial infarction. J Clin Invest. $1973 ; 52: 1569-1577$

4. Austin M, McKnight B, Edwards K, Bradley C, McNeely M, Psaty B, et al. Cardiovascular disease mortality in familial forms of hypertriglyceridemia: a 20-year prospective study. Circulation. 2000;101:2777-2782

5. Reiner Z, Catapano A, de Backer G, Graham I, Taskinen MR, Wiklund O, et al. ESC/ EAS Guidelines for the management of dyslipidaemias: The Task Force for the management of dyslipidaemias of the European Society of Cardiology (ESC) and the European Atherosclerosis Society (EAS). Atherosclerosis. 2011;271(Suppl 1):S1-S44

6. Stone N, Robinson J, Lichtenstein A, Merz C, Blum C, Eckel R, et al. 2013 ACC/ AHA guideline on the treatment of blood cholesterol to reduce atherosclerotic cardiovascular risk in adults: a report of the American College of Cardiology/American Heart Association Task Force on Practice Guidelines. Circulation. doi:10.1161 /01. cir.0000437738.63853.7a

7. Wierzbicki A, Humphries S, Minhas R. Familial hypercholesterolaemia: summary of NICE guidance. BMJ. 2008;337:a1095

8. Goldberg A, Hopkins P, Toth P, Ballantyne C, Rader D, Robinson J, et al. Familial hypercholesterolemia: screening, diagnosis and management of pediatric and adult patients: clinical guidance from the National Lipid Association Expert Panel on Familial Hypercholesterolemia. J Clin Lipidol. 2011;5(3 Suppl):S1-S8

9. Mata P, Alonso R, Ruiz A, González-Juanatey JR, Badimón L, Díaz-Díaz JL, et al. Diagnóstico y tratamiento de la hipercolesterolemia familiar en España: documento de consenso. Aten Primaria 2015 Jan;47:56-65

10. Mata P, Alonso R, Ruíz-Garcia A, Díaz-Díaz JL, González N, Gijón-Conde T, et al. Familial combined hyperlipidemia: consensus document. Aten Primaria. 2014 Oct; 46:440-446

11. Wiesbauer F, Blessberger H, Azar D, Goliasch G, Wagner O, Gerhold L, et al. Familial-combined hyperlipidaemia in very young myocardial infarction survivors $(<$ or $=$ 40 years of age). Eur Heart J. 2009:30:1073-1079

12. The ACCORD Study Group. Effects of combination lipid therapy in type 2 Diabetes Mellitus. N Engl J Med. 2010;362:1563-1574

13. Berglund L, Brunzell J, Goldberg A, Goldberg I, Sacks F, Murad M, et al. Evaluation and treatment of hypertriglyceridemia: An endocrine society clinical practice guideline. J Clin Endocrinol Metab 2012;97:2969-2989

14. Craig H. Make early diagnosis, prevent early death from familial hypercholesterolaemia. The MED-PED FH program. Med J Aust. 1995;162:454-455

15. Versmissen J, Oosterveer D, Yazdanpanah M, Defesche J, Basart D, Liem A, et al. Efficacy of statins in familial hypercholesterolaemia: A long term cohort study. BMJ. 2008;337:a2423.

16. Pijlman A, Huijgen R, Verhagen S, Imholz B, Liem A, Kastelein J, et al. Evaluation of cholesterol lowering treatment of patients with familial hipercholesterolemia: A large cross-sectional study in The Netherlands. Atherosclerosis. 2010;209:189-94

\section{Estatinas y otros Hipolipemiantes}

1. Baigent C, Keech A, Kearney P, Blackwell L, Buck G, Pollicino C, et al. Efficacy and safety of cholesterol-lowering treatment: prospective meta-analysis of data from 90,056 participants in 14 randomised trials of statins. Lancet 2005; 366(9493):1267-1278

2. Baigent C, Blackwell L, Emberson J, Holland L, Reith C, Bhala N, et al. Efficacy and safety of more intensive lowering of LDL cholesterol: a meta-analysis of data from 170,000 participants in 26 randomised trials. Lancet 2010;376:1670-1681

3. Byington R, Macfarlane P, Collins R, La Rosa J, Rossouw J, Probstfield J, et al. Efficacy and safety of LDL-lowering therapy among men and women: meta-analysis of individual data from 174000 participants in 27 randomised trials. Lancet. 2015;385:1397-1405

4. Mihaylova B, Emberson J, Blackwell L, Keech A, Simes J, Barnes E, et al. The effects of lowering LDL cholesterol with statin therapy in people at low risk of vascular disease: meta-analysis of individual data from 27 randomised trials. Lancet. 2012;380: 581-590

5. Kearney P, Blackwell L, Collins R, Keech A, Simes J, Baigent C, et al. Efficacy of cholesterol-lowering therapy in 18,686 people with diabetes in 14 randomised trials of statins: a meta-analysis. Lancet 2008;371:117-125

6. Palmer S, Craig J, Navaneethan S, Tonelli M, Pellegrini F, Strippoli GF. Benefits and harms of statin therapy for persons with chronic kidney disease: a systematic review and meta-analysis. Ann Intern Med 2012;157:263-275

7. Silva M, Swanson A, Gandhi P, Tataronis G. Statin-related adverse events: a meta-analysis. Clin Ther 2006;28:26-35

8. Preiss D, Seshasai S, Welsh P, Murphy S, Ho J, Waters D, et al. Risk of incident diabetes with intensive-dose compared with moderate-dose statin therapy. A meta-analysis. JAMA. 2011;305:2556-2564

9. Sattar N, Preiss D, Murray H, Welsh P, Buckley B, de Craen A, et al. Statins and risk of incident diabetes: a collaborative meta-analysis of randomised statin trials. Lancet. 2010;375:735-742

10. Peto R, Emberson J, Landray M, Baigent C, Collins R, Clare R, et al. Analyses of cancer data from three ezetimibe trials. N Engl J Med 2008;359:1357-1366

11. Pandor A, Ara R, Tumur I,Wilkinson A, Paisley S, Duenas A, et al. Ezetimibe monotherapy for cholesterol lowering in 2,722 people: systematic review and meta-analysis of randomized controlled trials. J Intern Med, 2009;265:568-580

12. SHARP Collaborative Group. Study of Heart and Renal Protection (SHARP): randomized trial to assess the effects of lowering low-density lipoprotein cholesterol among 9,438 patients with chronic kidney disease. Am Heart J. 2010;160:785-794

13. Cannon C, Blazing M, Giugliano A, McCagg A, White J, Theroux P, et al. Ezetimibe added to statin therapy after acute coronary syndromes. N Engl J Med 2015;375:2387-2397

14. The Lipid Research Clinics Coronary Primary Prevention Trial results. I. Reduction in incidence of coronary heart disease. JAMA 1984;251(3):351-64

15. Studer M, Briel M, Leimerstoll B, Glass T, Bucher H. Effect of Different Antilipidemic Agents and Diets on Mortality: A Systematic Review. Arch Int Med 2005;165:725-730

16. Rick M, Elo O, Haapa K, Heinonen O, Heinsalmi P, Helo P, et al. Helsinki Heart Study primary-prevention trial with gemfibrozil in middle-aged men with dyslipidemia. N Engl J Med 1987:317:1237-1245

17. Rubins H, Robins S, Collins D, Fye C, Anderson J, Elam M, et al. Gemfibrozil fo the secondary prevention of coronary heart disease in men with low levels of high density lipoprotein cholesterol. Veterans Affairs High-Density Lipoprotein Cholesterol Intervention Trial Study Group. N Engl J Med. 1999;341:410-418

18. Jun M, Foote C, Lv J, Neal B, Patel A, Nicholls S, et al. Effects of fibrates on cardiovascular outcomes: a systematic review and meta-analysis. Lancet 2010,375:1875-8

19. Dietary supplementation with $n-3$ polyunsaturated fatty acids and vitamin $E$ after myocardial infarction: results of the GISSI-Prevenzione trial. Gruppo Italiano per lo Studio della Sopravvivenza nell'Infarto miocardico. Lancet. 1999:354:447-455

20. Yokoyama M, Origasa H, Matsuzaki M, Matsuzawa Y, Saito Y, Ishikawa Y, et al. Effects of eicosapentaenoic acid on major coronary events in hypercholesterolaemic patients (JELIS): a randomised open-label, blinded endpoint analysis. Lancet. 2007 Mar 31;369:1090-1098

21. Kromhout D, Giltay E, Geleijnse J, Alpha Omega Trial Group. n-3 fatty acids and cardiovascular events after myocardial infarction. N Engl J Med 2010;363:2015-26

22. ORIGIN Trial Investigators, Bosch J, Gerstein H, Dagenais G, Díaz R, Dyal L, Jung $H$ et al. n-3 fatty acids and cardiovascular outcomes in patients with dysglycemia. $N$ Engl J Med. 2012;367:309-318 